\title{
Development of Tools, Instrumentation and Codes for Improving Periodic Examination and Repair of SFRs
}

\author{
François Baqué, ${ }^{1}$ Frédéric Reverdy, ${ }^{2}$ Jean-Michel Augem, ${ }^{3}$ and Julien Sibilo ${ }^{4}$ \\ ${ }^{1}$ Nuclear Technology Department, CEA, 13108 Saint Paul lez Durance Cedex, France \\ ${ }^{2}$ Imaging and Simulation for Control Department, CEA, 91191 Gif-sur-Yvette Cedex, France \\ ${ }^{3}$ EDF, SEPTEN, 12-14 Avenue Dutrievoz, 69628 Villeurbanne Cedex, France \\ ${ }^{4}$ Nuclear Power Division, AREVA, 10 rue Juliette Récamier, 69456 Lyon Cedex 06, France \\ Correspondence should be addressed to François Baqué, francois.baque@cea.fr
}

Received 21 December 2011; Accepted 1 March 2012

Academic Editor: Toshikazu Takeda

Copyright (๑) 2012 François Baqué et al. This is an open access article distributed under the Creative Commons Attribution License, which permits unrestricted use, distribution, and reproduction in any medium, provided the original work is properly cited.

In the frame of the CEA, EDF, and AREVA coordinated research program launched in 2007 for the development of Generation IV sodium-cooled fast reactors (SFRs), the improvement of in-service inspection and repair (ISI\&R) capabilities was identified as a major issue. Within the French-associated multiannual SFR research program, the periodic examination and repair are looked at through the following main R\&D axes: (i) improvement of the primary system conceptual design in order to ease periodic examination and repair, (ii) development of inspection techniques (periodic inspection tools and associated simulation), (iii) accessibility and associated robotics, and (iv) development and validation of repair processes. Associated needs are being defined through an iterative method between designers and inspection specialists: adaptation of the SFR design to ISI\&R requirements, validation of the ultrasonic transducers, of associated ultrasonic nondestructive examination techniques, of laser repair processes, of associated robotic equipment. International collaboration is also running for some specific items such as ultrasonic visualization under liquid sodium.

\section{Introduction}

From within the framework of the French Act, dated June 28, 2006, which requests an assessment of the industrial perspectives of transmutation by 2012, the Generation IV systems, especially the sodium-cooled fast reactors seem to be the most mature technology to be developed among the systems proposed for sustainable energy. Thus, since 2007, the CEA, EDF, and AREVA French partners launched a coordinated research program on sodium cooled fast reactors (SFR), which is now being used for the next ASTRID (advanced sodium technological reactor for industrial demonstration) prototype [1]. These program main axes were classified into four R\&D items:

(1) design of a core with enhanced safety;

(2) better resistance of SFRs to severe accidents and external hazards;
(3) research on an optimized energy conversion system to reduce the sodium risk;

(4) review of reactor and component design options in order to:

(i) improve inspection, maintenance, availability, and decommissioning;

(ii) reduce the environmental impact while reinforcing resistance to proliferation;

(iii) improve the reactor performance and overall economic aspect.

Among these items, improvement of in-service inspection and repair (ISI\&R) is a major transverse issue [2]. ISI\&R is strongly linked to safety analysis (the three defense lines: checking the state of material and equipment during the reactor's life span, detection of premature failures, and in 
operation detection of significant failures), economic reliability (implementation delays), and investment protection (repair).

One of the major difficulties for SFR ISI\&R deployment is the sodium environment. Indeed, the sodium has several characteristics which tend to make in-service inspection and repair complex, especially by comparing with Light Water Reactor ISI\&R.

Firstly, the sodium is very reactive with air and water: this leads to monitor carefully the confinement and steam generators leakage and to control the tightness during the interventions.

Secondly, due to the risk of under stress caustic corrosion appearance (when a small quantity of sodium, recovering the stainless steel internal structures is in the presence of moisture), the reactor sodium draining is only planned for exceptional interventions, and the current inspections and operations have then to be realized under sodium.

Thirdly, linked to the sodium physical characteristics, the temperature corresponding to plant cold shutdown condition is about $200^{\circ} \mathrm{C}$ (the in-operation temperatures being comprised between $400^{\circ} \mathrm{C}$ and $550^{\circ} \mathrm{C}$ ). Thus, ISI\&R devices have to work at these high temperature levels.

Last but not least, the sodium being opaque, each intervention (inspection/repair) below the free sodium level cannot be performed/followed by optical devices, so, up to now the mostly used techniques are the ultrasonic ones.

Considering the ISI\&R importance for SFR, but also the sodium environment complexity, the multiannual R\&D program launched in 2007 takes into account feedback experiences from former French sodium reactors (Rapsodie, Phenix and Superphenix [3]).

This specific R\&D program, whose global objective is to increase ISI\&R capabilities, can be divided into four sections [4]:

(i) conceptual design improvement of the primary and secondary systems in order to reduce the number of structures and components to be surveyed, to locate sensitive zones in accessible areas from either inside or outside, and to reduce the number and length of welds;

(ii) development of measurement techniques and surveillance devices for continuous monitoring during reactor operation and for periodic examinations during reactor shutdown;

(iii) improvement of accessibility and development of remote-controlled devices (robotics) with suitable carriers;

(iv) identification, development, and validation of repair processes (core discharge and sodium draining), as well as techniques for repair operations in sodium environment.

Based on existing ISI\&R feedback experience from sodium-cooled systems, the general ISI\&R objectives were defined, with respect to the preliminary specifications issued by the French utilities (EDF) for the future SFRs.

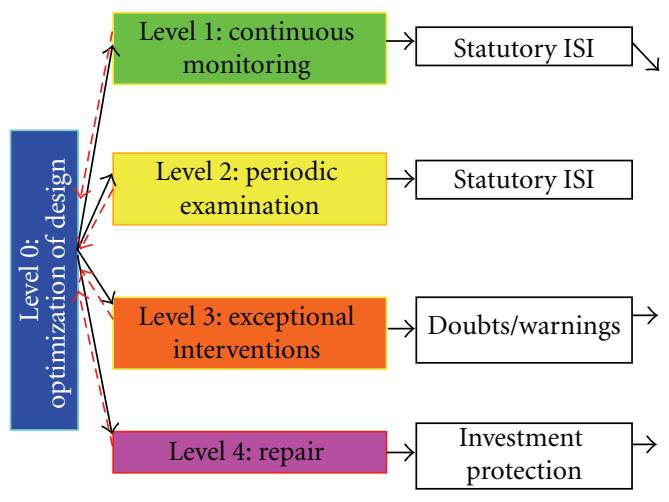

FIGURE 1: ISI\&R approach for future French SFRs.

For ASTRID first criticality, it will be necessary to get a sufficient, available, efficient, and qualified instrumentation. Moreover, it is planned to test in ASTRID during its operation, the instrumentation which has to be qualified for the future nuclear plants (Generation IV).

The running program of research and development implies, on the one hand, usual and available devices to be improved, and, on the other hand, innovative ones to be developed, tested, and qualified.

This paper focuses on the French developments undertaken for periodic examination and repair of reactor core vessel and associated internals. ISI\&R of other components (such as heat exchangers like steam generator units) are also looked at in the running program but are not described here.

\section{Specifications for ISI\&R Future SFRs}

In service inspection for SFR must take part in the reactor integrity demonstration, by checking the structures insuring the three main safety functions (reactivity monitoring, decay heat removal, and containment of hazardous products).

The surveillance graduation applied to each structure is defined thanks to the "risk-informed method," which takes into account the damage probability all along plant life and the associated consequences.

Repair ability is also an important specification for SFR, as it can save the overall investment.

ISI\&R approach is now parted in five levels for SFR design (Figure 1): a new Level 0 allows both to reduce ISI\&R needs by design optimization and to take into account ISI\&R requirements for design.

2.1. L1-Continuous Monitoring. ISI\&R level 1 deals with continuous monitoring during reactor operation (including power operation with sodium temperature reaching $550^{\circ} \mathrm{C}$ ). It is mainly based on the operating parameters checking (neutronics, temperatures, flow rates, and pressure) with some global measurements reflecting the structure and component states (leaks, mechanical deformation, and vibratory or acoustic characteristics). continuous monitoring of the inner structures within the reactor block (Figure 2) is mainly performed by the book keeping of reactor phases 


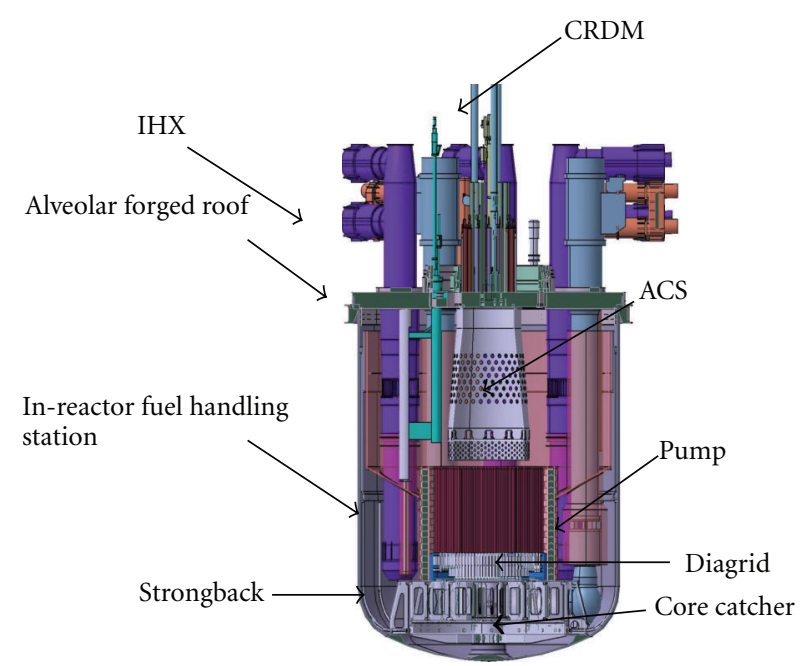

Figure 2: Preconceptual design for SFR reactor block.

(countdown of allowed transients). Level 1 monitoring has to early detect any parameter drifts, or irregularities with regard to the operating range. This item is out of the scope of the present paper.

2.2. L2-Tests and Periodic Examinations. During reactor cold shutdowns (sodium at about $200^{\circ} \mathrm{C}$ ), ISI\&R level 2 brings together tests and examinations in a statutory program (NDE purpose: nondestructive examinations).

In addition to the confirmation of the status observed by continuous monitoring, the periodic inspection program must provide the validation of the hypothesis and project values about the damaging mechanisms considered during the design studies. Moreover, ISI\&R level 2 must look for the unforeseen damaging mechanisms, in order to check the material mechanical property degradation process, and the absence of corrosion-type mechanism. Mainly focused on safety-related structures (core support structure (see Figure 3), above core structure (ACS), and reactor vessel), these checks may be also carried out on minor structures (inner vessel, and various baffles).

Even if some components can be inspected after their removal from the reactor (primary pump (PP), control rod drive mechanisms) for maintenance, other parts of the reactor block need specific means for in situ inspection.

Because they are easier and quicker, other inspections are preferentially performed from outside the main vessel, even for example, using immersed structures as ultrasonic waveguides.

Nevertheless, other inspections have to be performed within the reactor block: the checks mainly consist in visual observation in the gas upper volume, but also in sodium telemetric positioning control, and volumetric non-destructive examinations of welded junctions.

Even if up to now ultrasonic techniques are mainly used, other means are of course to be investigated.

Although the SFR inspected zone rate has to be highest as possible; nevertheless, the periodic inspection program
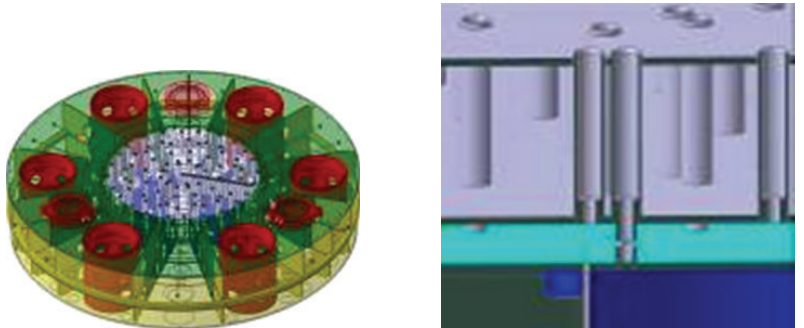

FIGURE 3: Integrated diagrid-strongback option (general view and strongback access detail).

duration must be suitable for a high-target plant availability rate.

2.3. L3-Inspection Exceptional Extension for Analysis. ISI\&R level 3 is the answer to abnormal situations, which may be consecutive to in-operation warning or to an operation mistake. This ISI level purpose is to allow exceptional intervention for control. From reactor shutdown, due to unforeseen event, to the localization and characterization of the fault, the level 3 insures the entire range of associated operations.

According to the possible hazardous situations, this level must enable to reach and inspect most of the reactor zones. For that purpose, it is necessary to provide the most extensive access to the internal structures. First, the design studies have to take this requirement into account. Then, the sodium-immersed devices, including sensors and carriers (like for L2), have to be available. Furthermore, if the needed operation requires a more accurate examination, or if it is sodium incompatible, core unloading and sodium draining have to be performed. These provisions, considered only in exceptional cases, are studied since the preliminary $R \& D$ phase. Main issues deal with draining without caustic stress corrosion risk and performing the entire operation (core unloading, draining, examination, and reverse operations) within the duration target of one year maximum.

In addition, the primary components withdrawal (intermediate heat exchangers IHX, PP) may be necessary to get an improved access. The target duration for the associated removal and replacement is about one month per component (within the one-year maximum duration for exceptional intervention).

In this frame, only few technical solutions are available, then this justifies undertaking a significant effort for innovation, especially for under-sodium applications.

2.4. L4-Repair and Replacement. For SFRs, it is necessary to be able to guaranty the investment protection during the whole life span, even in case of unforeseen damage. That is why ISI\&R specifications incorporate the repair ability for all the structures, and the replacement possibility for the primary components. These provisions are now insured by ISI\&R level 4.

The level 4 operations are considered, when the defect origin has been identified (after levels 2 or 3 examination). 
Repair or replacement operations are performed in sodium environment: in the argon cover gas standard volume, under sodium with tight cavity, or in argon gas after sodium draining. The choice between the repair and the replacement is made by taking into account the damaged structure type (replacement possibility), the failure extent, and the implementation costs and durations.

To favor this ISI\&R level implementation, the reactor structures have to be simple and accessible. As far as possible, the high thermomechanically loaded structures have to be designed as replaceable. Then, the ISI\&R level 4 operations require repair tools to perform preliminary sodium local removing, machining, and welding. The robotic carriers, which carry the tools, have to be suitable for sodium environment, but they have also to absorb machining forces, and to recover the produced wastes.

Of course, repair has to be avoided; nevertheless, associated operations have to be foreseen. Consequently, both reactor block and repair tools have to be designed in adequacy in order to allow any exceptional intervention.

Even if the main challenge deals with the reactor block system, the same approach is also applied to all other sodium devices and loops.

In this frame, other useful existing industrial or $\mathrm{R} \& \mathrm{D}$ feedback experience is looked at, whatever the original field.

\section{Design Studies for ISI\&R Improving}

3.1. Global Design. Being a welded stainless steel manufacturing, operating at high temperatures $\left(400-550^{\circ} \mathrm{C}\right)$, with significant thermal gradients, under neutron and gamma fluxes, during 60-year lifetime, future SFRs will undergo some significant loadings. These loadings may lead to failure modes such as:

(i) excessive deformation;

(ii) ratcheting;

(iii) fatigue for the cold pool zones;

(iv) creep-fatigue for the hot pool zones;

(v) buckling for thin structures in compression;

(vi) thermal striping for zones subjected to high thermal gradient mixtures;

(vii) irradiation damage for the structures near the core;

(viii) corrosion;

(ix) wear.

The risk reduction of the potential damage, which is an important issue for design studies, also allows minimizing surveillance, examination, and repair needs. Thus, the reactor global design has to be selected, taking into account this requirement, and by collaborating with ISI\&R specialists. The work mainly consists in proposing a robust design with no thermomechanical weak areas. Therefore, the welds number has to be reduced, and the remaining ones are to be located in low-loaded areas. Moreover, geometrical singularities which may induce some stress concentrations or unusual operating ranges (cavitation wear, erosion, excessive, and vibrations) are to be avoided. Finally, this previous design work, which reduces ISI\&R provision range, is being made for SFR design architecture (1500 MWth pool type) shown in Figure 2.

A related work is undertaken by the French partners for improving existing design rules: thus, RCC-MRx new code will include, in 2012 edition, NDE "good practice" information in order to ease/make them possible, through specific NDE recommendations for designers. This is part of Level 0 item (see Figure 1).

3.2. Access and Examination Ability Improvements. The reactor design has to favor ISI implementation with the help of sufficient accessibility and a suitable positioning of areas to be inspected. Therefore, an advanced design of the pool type architecture has been proposed. Additional design studies have to be made to fully increase the reactor design ability for ISI\&R deployment and performing.

3.3. Repair and Replacement Ability. The design has also to deal with repair operations by simplifying the structures and with replacement operations enabled by the removable component proposals.

The structure simplification has been evoked in the previous paragraphs, and the design proposed options contribute to make the repair operations easier, especially the ISI\&R dedicated pool type reactor with few and simple internal structures.

The replacement operations have also to be foreseen since the design phase, taking into account the components and structures which may require it. Being the case for the PP and the IHX, some optimizations of the interfaces between the components and the roof have been proposed in order to make the removal easier and to reduce the associated durations. Above core structure (ACS) is a highly thermomechanically loaded structure, and so its replacement during reactor lifetime has to be considered too.

\section{Technological Developments}

4.1. Multiannual R\&D Program. In parallel to increasing ISI\&R performances, the feedback experience taken into account, and the design improvements, the new SFR ISI\&R objectives require also some technology progresses, and more particularly for under sodium devices: non destructive examination (NDE), tools for basic repairs, robotic for carriers.

Thus, a multiannual R\&D program, implying the CEA, EDF, and AREVA, started in 2007 for SFRs, the following paragraphs focusing on the main significant developed technologies up to now in this frame.

Since 2010, the program purpose is not only to develop and to master the ASTRID ISI\&R requested technologies but also more innovative ones.

\subsection{Inspection Simulation: CIVA Software for Ultrasonic Applications. Ultrasonic inspection deals with:}


(i) telemetry measurement within primary sodium of the main vessel, for the periodical checking of internal structure location;

(ii) nondestructive Examination of internal structures and vessels.

Two approaches are being followed: inside inspection where transducers are directly immersed in sodium coolant (see Figure 8 and Section 4.3) and inspection from outside with transducers positioned along the wall of the main vessel (see Figure 4 and Section 4.4). For both cases, acoustic bulk waves and guided/surface waves (see Figure 12 and Section 4.5) are looked at. Probe design and inspection performances can be predicted by using comprehensive models that can take into account the various variables of the problem: attenuation and deflection, transmission and reflection of ultrasounds, which depend on liquid sodium characteristics (mainly temperature and micro bubble fields), of immersed structures characteristics (mainly wetting at liquid/solid interface, thickness, and shape).

\subsubsection{Simulation of Ultrasonic Inspection Through Screens.} Telemetry based on ultrasonic waves can be used to monitor the position of internals and equipment introduced inside the vessel. Ultrasonic probes positioned outside the reactor along the main vessel wall can be used to perform telemetry measurement without requiring previous sodium conditioning (Figure 4). However, inspection from outside requires to go through one or several metal plates (main vessel wall, baffle) leading to losses of energy at each interface. It is important to determine the level of these losses in order to estimate the possibility to measure the position of the various internals and propose other configurations of inspection in case the signal-to-noise ratio (SNR) becomes too low. One way to perform this estimation is to use modeling.

Ultrasonic simulation tools have been developed and implemented in CIVA $[5,6]$. A method based on a pencil approach allows the calculation of ultrasonic beam fields in heterogeneous media taking into account material attenuation and energy losses at interfaces between media of different acoustic properties. Kirchhoff models have been developed to calculate the interaction of these beams with surfaces and/or defects of various shapes. In order to validate these models for telemetric applications, experiments were carried out in water with two $40 \mathrm{~mm}$ thick stainless steel plates supposed to represent the main vessel wall, and an internal structure was used. Transducers were positioned along one of the plates in a pulse/echo normal configuration and in a pitch and catch nonnormal incidence configuration.

For normal incidence application, CIVA predicts well the exponential decay of the ultrasonic energy reflecting back and forth in the plate supposed to represent the main vessel wall (Figure 5). Multiple reflections still have significant energy by the time the echo off the front surface of plate 2 is detected, meaning that it can be difficult to interpret echoes coming from objects located close to the main vessel wall. Simulation can thus be used as a tool to predict the arrival times of the various echoes reflected off the internals. CIVA predicts correctly the amplitude of all the echoes,

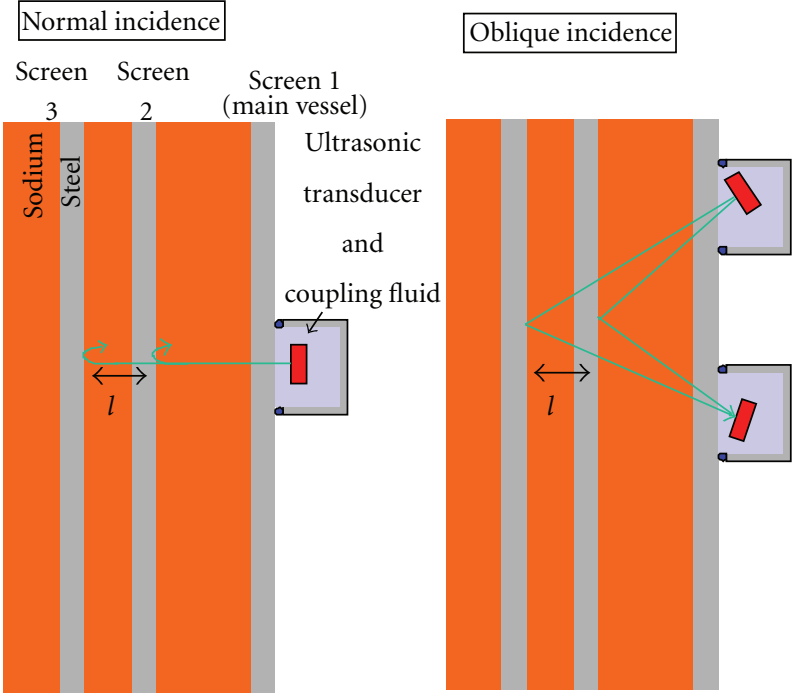

FIGURE 4: Inspection through screens, for a normal incidence with a transducer working in pulse/echo mode (left) and nonnormal incidence with a pitch-catch configuration (right).

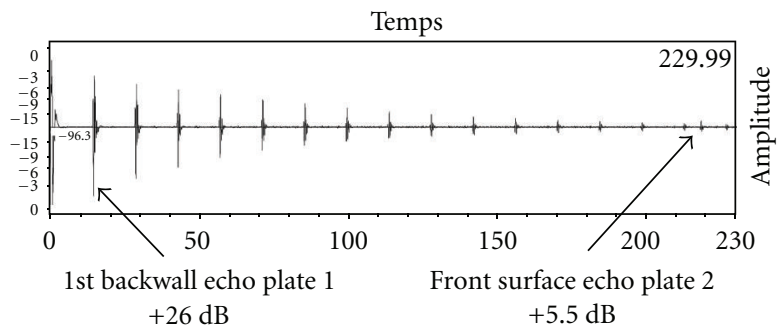

(a)

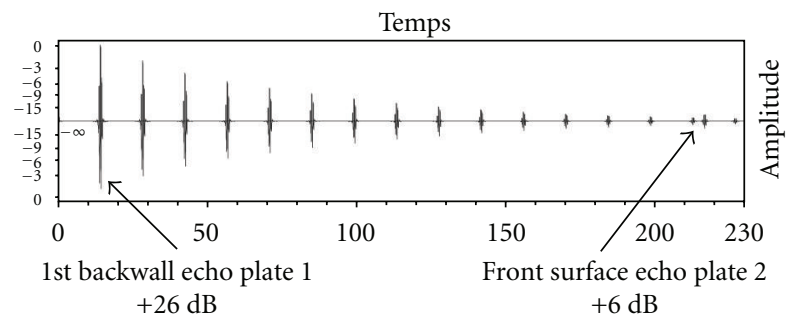

(b)

FIGURE 5: Inspection through screens for a normal incidence configuration at $2.25 \mathrm{MHz}$, experimental (a) and simulation (b).

particularly the echo coming from the front of the surface of the second plate supposed to represent the surface of an internal structure.

For non-normal incidence application (pitch and catch configuration with ultrasonic waves propagating at $45^{\circ}$ in the main vessel wall), the distance between the emitter and the receiver is adjusted to obtain an intersection of the emission and reception focal paths along the surface of plate 2. Non-normal configurations can be used to inspect internals that are not parallel to the main vessel wall and/or to decrease the duration of the multiple echoes inside the main vessel wall observed at normal incidence. When comparing 


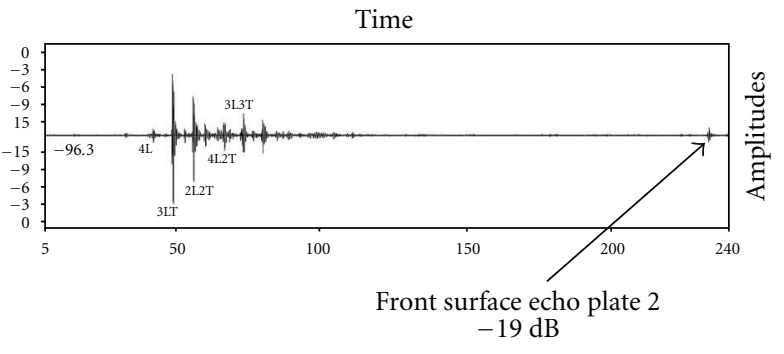

(a)

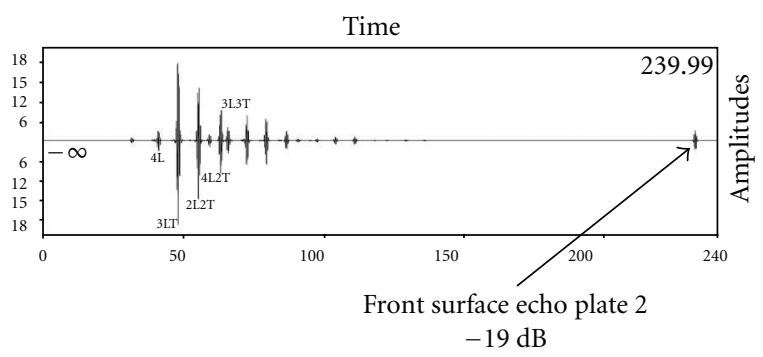

(b)

FIGURE 6: Inspection through screens for a $45^{\circ}$ configuration at 2.25 MHz, experimental (a) and simulation (b).

experimental results and simulation calculated with CIVA (Figure 6), we observe multiple echoes early in the signals. They are coming from modes converted inside the main vessel wall; their time of flight allows analyzing each of them. The amplitude of the front surface echo off plate 2 is measured; reference is the mode conversion echo noted $3 \mathrm{LT}$.

We see that CIVA predicts correctly the mode conversions obtained in the first plate representative of the main vessel wall. We confirm that the duration of these echoes is shorter than the multiple echoes observed for the normal incidence configuration, allowing the detection of objects closer to the main vessel wall. We verify as well that the amplitude and the time of flight of the echo obtained off the surface of the second plate are well predicted.

Validations performed for various frequencies and various angles of incidence confirm that CIVA can be used as a tool to predict telemetric applications for a quasistatic fluid. Further validations will be conducted for configurations with a third plate representative of a baffle and with defects inside the plate supposed to represent the internal. This will help determining which internal can be inspected from outside the main vessel wall.

4.2.2. Simulation of Ultrasonic Inspection in Turbulent Fluid. Models presented in the previous section are valid for homogenous fluids meaning quasi-static and isothermal fluids: sodium is likely to be in that state during shutdowns for periodic inspections. For measurements associated to reactor operation, the flow of sodium creates zones of turbulence, which induce local variations of temperature. These variations translate into velocity inhomogeneities, which can impact greatly the precision of telemetry measurements.
It is thus important to have the capability to predict the influence of the turbulence on the precision of the telemetry measurements.

Telemetry inside the reactor can be performed to monitor core compaction for example. A probe positioned at the core exit would see effects of sodium flow, which can create local variations in temperature of approximately $50^{\circ} \mathrm{C}$ that translate into velocity variations of $1 \%$ (see next formula). We have followed two approaches to model the propagation of ultrasonic waves in a inhomogeneous fluid: a determinist model that propagates rays inside a predetermined cartography representative of the temperature distribution and a statistical model that modifies times of flight according to a distribution representative of the inhomogeneities inside the fluid.

We first generate cartography of temperature, which is going to be used as the medium of propagation. The generation of the cartography is based on a Gaussian model of thermal turbulence [7]. The parameters of the model are the maximum temperature variation and the characteristic length $L$, which represents the scale of the inhomogeneities. This technique allows generating spatial temperature cartographies from which we generate spatial velocity cartographies using the following basic relationship:

$$
c(\mathrm{~m} / \mathrm{s})=2577.2-0.5234 \mathrm{~T}\left({ }^{\circ} \mathrm{C}\right),
$$

where in sodium sound velocity $c$ depends only on sodium temperature $T$.

After building the medium of propagation, we apply the ray theory to model the acoustic field. The process is done in two steps: the first one calculates the time of flight between a point source and a point of observation solving a system of differential equations known as the kinematic ray tracing system. The second step allows the calculation of the amplitudes associated to the rays determined in the first step. Calculation at one point is based on the energy conservation criterion in a tube of rays.

We used the model to calculate the acoustic rays radiated by a punctual source for different values of the characteristic length $L$ supposed to represent the inhomogeneities met in a sodium-cooled reactor in operation. The $L$ value depends on the specific case to be treated: for example, at core outlet, $L$ should corresponds to subassembly spacing. The results are shown in Figure 7; the rays appear in black and are superimposed to the velocity cartographies calculated with each characteristic length.

We see that rays are not affected in terms of direction except for the case $L=50 \mathrm{~mm}$ (small inhomogeneities). Variations of time of flight are smaller than $4 \%$, and there is phase continuity between neighbour rays. It is necessary to take into account the variations of time of flight to consider the interferences between neighbour rays. We noticed as well small variations in amplitude (less than $0.1 \mathrm{~dB}$ ) compared to the propagation in a homogeneous medium meaning that we do not need any amplitude correction.

The goal of the statistical aberration model is to estimate the spatial variations of the time of flight of an acoustic beam after propagation into a turbulent medium. Using 


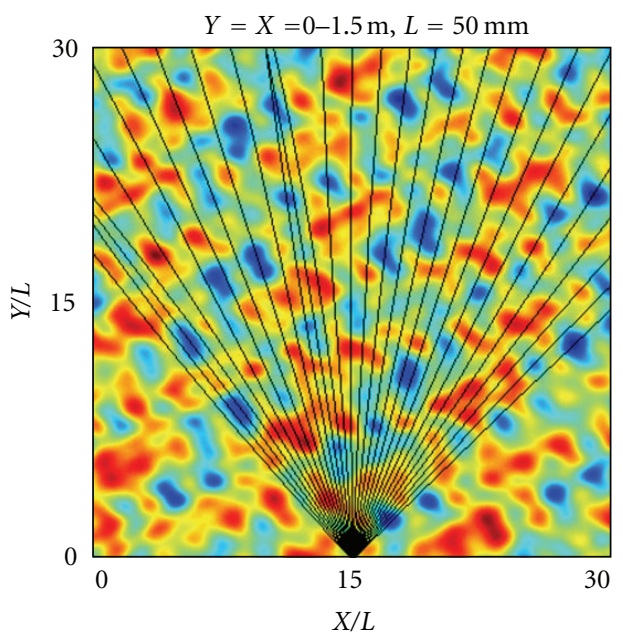

(a)

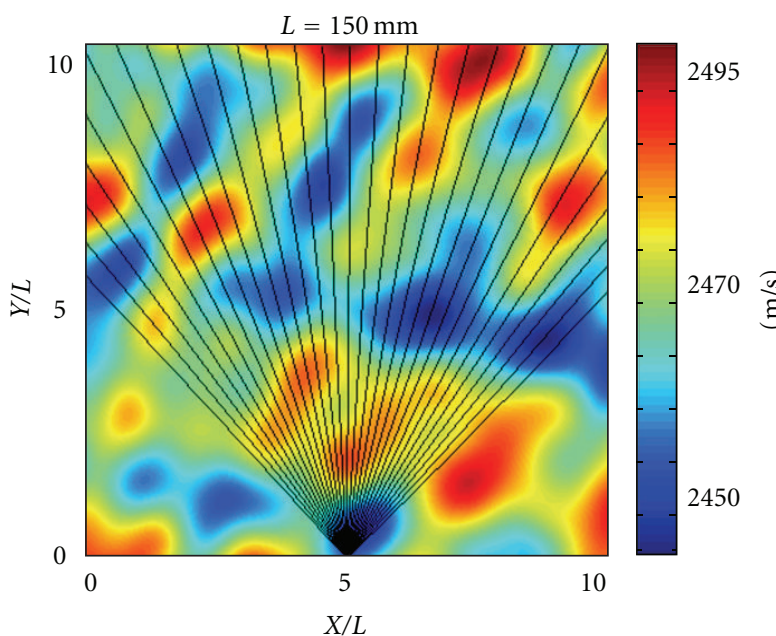

(c)

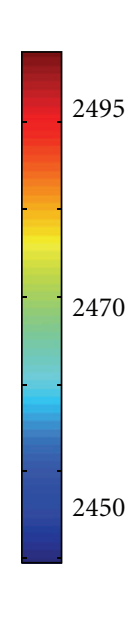

495
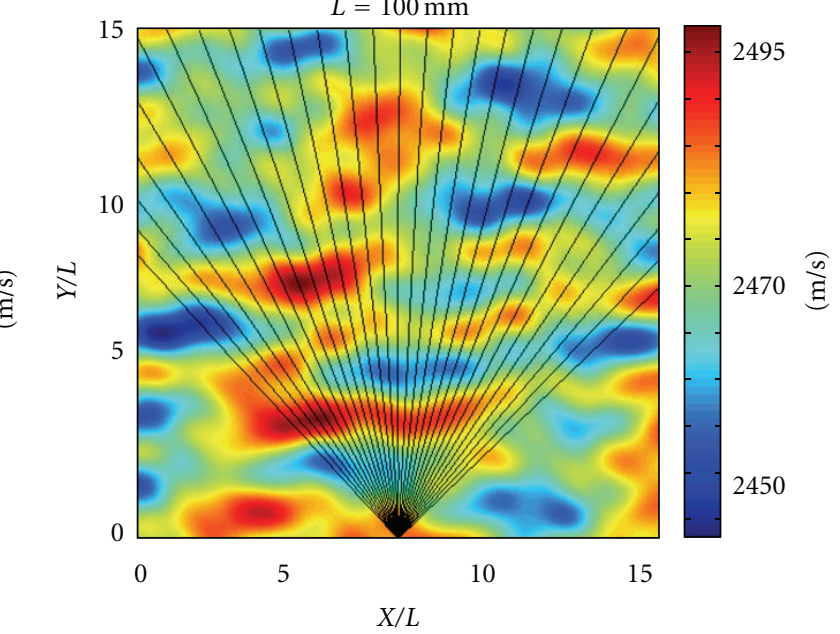

(b)

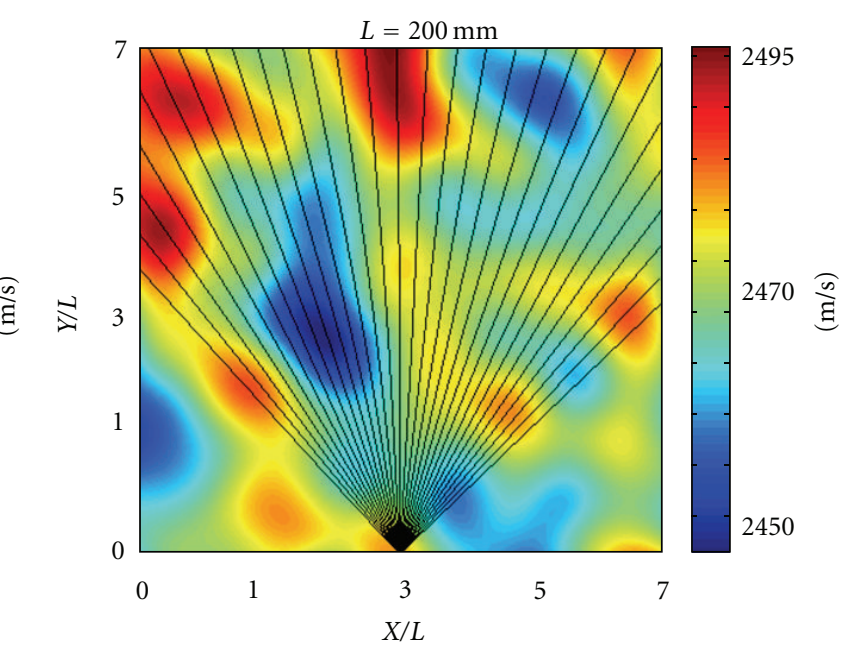

(d)

FIGURE 7: Ray tracing using the determinist model for four different values of the characteristic length.

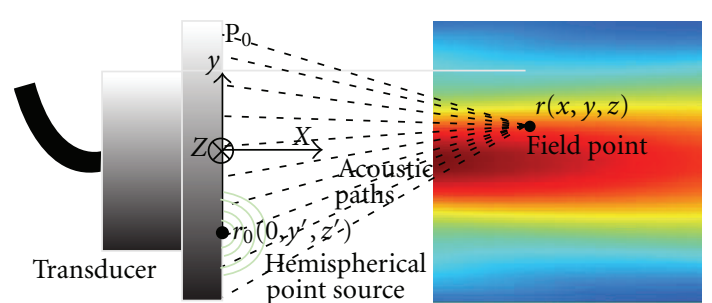

FIGURE 8: Ultrasonic transducer with radiating surface, in sodium acoustic beam predicting.

these variations, we introduce a time correction to the model used for homogenous media to represent the inhomogeneity of the medium. The statistical aberration model is based on a stochastic process: it generates a series of spatially continuous random values. We thus need statistical inputs such as the average time of flight, the time of flight variance, and a correlation function to insure spatial phase continuity.
The average and variance values can be obtained from the determinist model by realizing a great number of velocity cartographies for the same characteristic length. The correlation function is as well extracted from the determinist model results obtained for a point source and observation points located at equal distance to the source.

We applied the two models to calculate the acoustic beam field radiated by a transducer into an inhomogeneous fluid. A transducer can be modelled by a distribution of particle velocity source over the radiating surface of the transducer. A Rayleigh integral describes the acoustic scalar potential at any observation points caused by a set of sources located on the radiating surface. This formulation considers that each elementary source (a hemispherical point source) on the radiating surface contributes to the field at an observation point with amplitude inversely proportional to the acoustic path between the two points. Then the field is computed by a simple integral over the whole radiating surface of these contributions (Figure 8). 


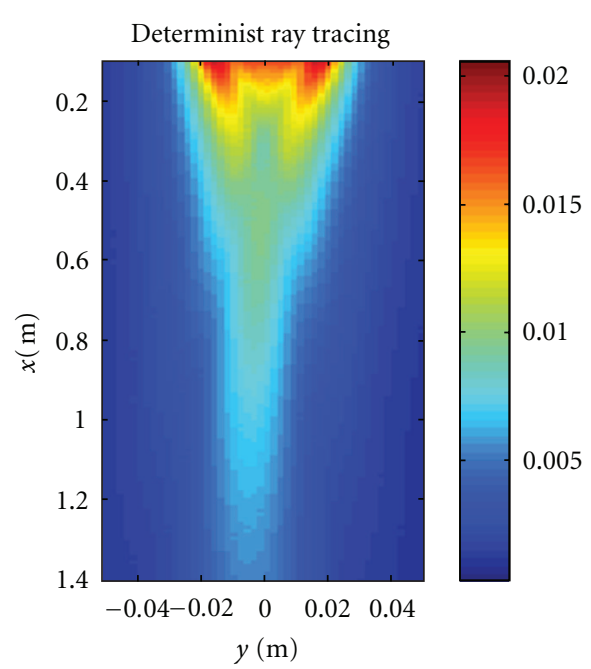

Figure 9: Acoustic beam propagation in inhomogeneous medium calculated by determinist ray tracing model (acoustic source position is at $[x=0 ;-0.02<y<0.02]$ coordinates).

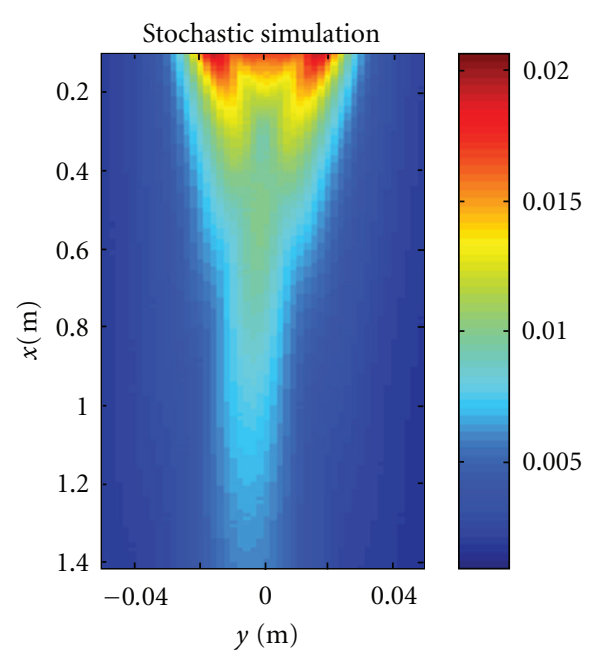

FIGURE 10: Acoustic beam propagation in inhomogeneous medium calculated by stochastic simulation mode (acoustic source position is at $[x=0 ;-0.02<y<0.02]$ coordinates).

Figure 8 shows the acoustic field calculated in a homogeneous medium. The integral is calculated over a circular radiating surface of $30 \mathrm{~mm}$. The signal is a Gaussian-modulated sinusoidal pulse with a central frequency of $2 \mathrm{MHz}$ and a $60 \%$ bandwidth. To calculate the acoustic beam in an inhomogeneous medium, we compute the acoustic paths using the determinist model for a given inhomogeneous velocity cartography. The impulse response for each point is the convolution of all contributions from the radiating surface. Figure 9 shows an example of the acoustic beam prediction in an inhomogeneous medium calculated by the determinist ray tracing. The parameters of the transducer are the same as the ones used for the homogeneous case. The random velocity is defined by the standard deviations $\sigma_{\varepsilon}=0.0018$ and the characteristic length $L=100 \mathrm{~mm}$.

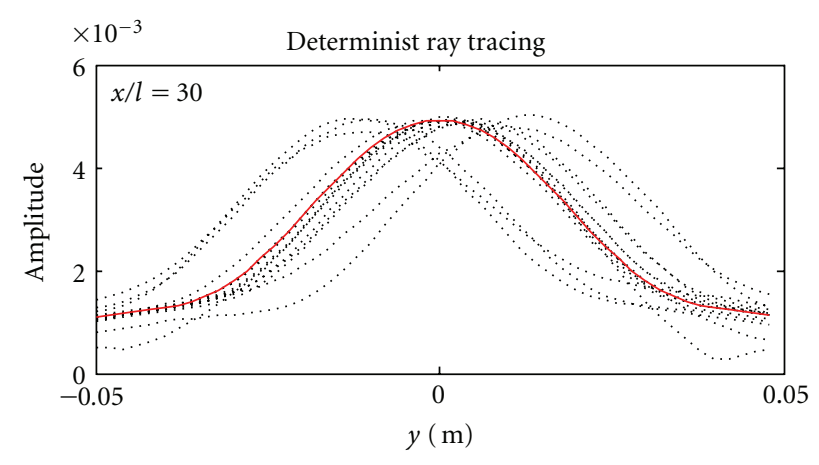

(a)

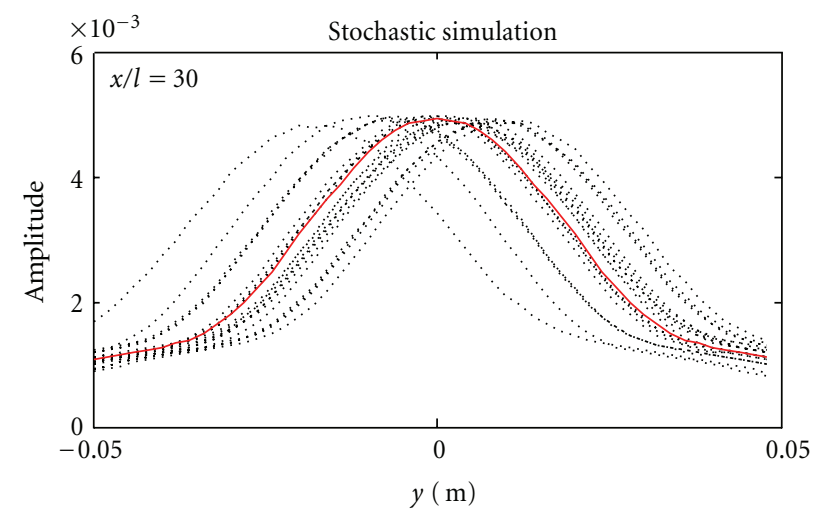

(b)

Figure 11: Acoustic beam deviations for the determinist and stochastic models; result for the homogeneous model is in red.

We find that the focal axis of the acoustic beam is no longer aligned with the normal to the surface of the transducer. The inhomogeneities lead to some slight deviations of the acoustic beam. At a distance of $1.4 \mathrm{~m}$, we observe a deviation of $0.01 \mathrm{~m}$, which means that the beam could totally miss the intended target and hit a different location. We then calculated the beam field using the statistical aberration model. The field is first calculated for a homogeneous medium by the Rayleigh integral, and the stochastic simulation model provides the variations of travel time for all acoustic paths. Result is displayed in Figure 10.

We can see that the acoustic beam predicted by the stochastic model also displays a deviation off the main focal axis similar to the one observed with the determinist model. To compare the two models, we ran several calculations for both models and compare the deviation to a calculation for a homogenous medium. Results are displayed in Figure 11; the red curve represents the result for the homogenous case and the dotted curves represent the result of each calculation for each model. Acoustic field is calculated for a single given random map of insodium sound velocity.

We see that the deviations obtained with both models are similar. However, the computation time of the determinist model is proportional to the number of rays, the number of observation points, and the discretization of each path. It is thus much more time consuming than the statistical model. In the future, we will use the stochastic approach to 


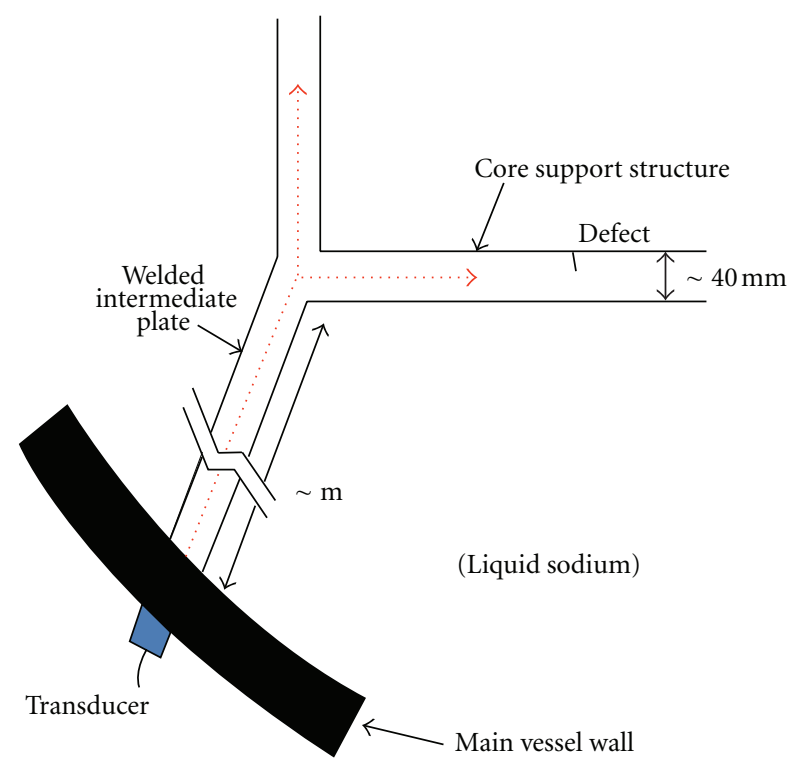

FIGURE 12: Inspection of structures used as wave guides.

predict the influence of the flow turbulence on the detection of internals during operation.

4.2.3. Simulation of Ultrasonic Inspection with Guided Waves. We expect to examine structures welded to the main vessel wall such as the core support, using guided waves. The goal is to generate guided waves from the main vessel external skin up to the inner vessel zone to control, by using intermediate plates as wave guides (Figure 12). The CEA has implemented in CIVA a model for the propagation of elastic guided waves using the semianalytical finite element method (SAFE) $[8,9]$. This method allows predicting the elastic field under the form of a modal decomposition for guides of complex section. It decomposes the solution into an analytical part for the propagation of the waves along the guide axis and a numerical approach using finite elements for the section of the guide. This method is, however, limited to guides that have a constant section along one extrusion plane and to defects that are contained along the section of the guide.

To model the propagation of guided waves along the core support, we need to be able to deal with discontinuities such as the junctions between the various parts of the core support and defects of arbitrary shapes. One way to handle these problems is to use a finite element method around the areas presenting these discontinuities. Diffraction by a discontinuity or a defect is calculated using the finite element (FE) method to which we add transparent boundary conditions allowing to minimize the volume meshed (Figure 13).

4.3. Ultrasonic Control with Sodium-Immersed Sensors. For future SFRs, ultrasonic transducers working under sodium at about $200^{\circ} \mathrm{C}$ are required [10]. They are planned to be used for the telemetric and volumetric examinations of the internal structures, during periodic inspection phase. If needed, they also could be exceptionally used for the core support structure. These transducers must be able to measure distance of some meters with $100 \mu \mathrm{m}$ accuracy and to detect cracks of about $200 \mathrm{~mm} \times 10 \mathrm{~mm}$.

On the one hand, with regard to the high-temperature transducers, these volumetric examination transducers are operated at a lower temperature and during a shorter duration. It implies a constraint reduction for the electroacoustic element choice. On the other hand, this kind of transducer use demands a better damping and efficiencies than hightemperature transducers and easier focusing capacities. Furthermore, the low temperatures (below $300^{\circ} \mathrm{C}$ ) may cause some acoustic coupling deficiency, hence, requiring a special treatment of the transducer active face.

4.3.1. Ultrasonic Transducers for Control in Liquid Sodium. Available Standard high temperature ultraSonic transducer (TUSHT) has been qualified by CEA in sodium and used in Phenix plant during 15 years: it is a multipurpose transducer, that was initially designed in order to be usable in a wide range of frequency (up to $5 \mathrm{MHz}$ ) and of applications, in the main vessel of sodium-cooled fast breeder reactors (and also PWR reactors), for all reactor conditions (shutdown, full power, and transients).

It uses a piezoelectric lithium niobate single crystal, $\mathrm{LiNbO}_{3}$ or $\mathrm{LN}$, with a Curie temperature of approximately $1150^{\circ} \mathrm{C}$.

The casing is made of AISI 304L stainless steel. The transmission of ultrasonic signals through the front face of the casing is ensured through an efficient acoustic bonding between the casing and the crystal, via a hard-soldering technique, developed by CEA. This bonding allows stable high-frequency transmission (up to $5 \mathrm{MHz}$ at least), at hightemperatures (more than $550^{\circ} \mathrm{C}$ ).

Two models have been qualified: TUSHT 4540 (LN diameter: $40 \mathrm{~mm}$; overall diameter $55 \mathrm{~mm}$ ) and TUSHT 4515 (LN diameter: $15 \mathrm{~mm}$; overall diameter: $32 \mathrm{~mm}$ ). Front faces are flat, or concave (spherical or cylindrical) to focus the ultrasonic waves. Figures 14 and 15 show a drawing of the TUSHT architecture and examples of TUSHT 4540 realizations.

The standard TUSHT (Figure 15(a)) is built with a front face thickness which must be compatible with very long service time (30 years) at full-power conditions. The frequency response contains several resonance frequencies (ranging from $0.7 \mathrm{MHz}$ to $4.5 \mathrm{MHz}$, approx.). Ultrasonic pulses rise times (5-15 $\mu$ s, approx., according to frequency) and durations are compatible with telemetry purposes (overall accuracy of $0.1 \mathrm{~mm}$ can be achieved, with use of suitable detection processing), but may be too long with regards to NDE usual specifications (e.g., flaw detection).

The immersed TUSHT are, at the present time, reference transducers for use in the main vessel of sodium fast reactors, in all the possible operating conditions. The major current improvement studies aim at reducing the oxygen-loss causes and effects (it is why one can see in Figure 14 two so-called "breathing tubes" which allow continuous air supply within the casing), for use at full-power conditions $\left(550^{\circ} \mathrm{C}\right.$, long time operation), and to improve the bandwidth in pulseecho applications, at least for use in inspection conditions 


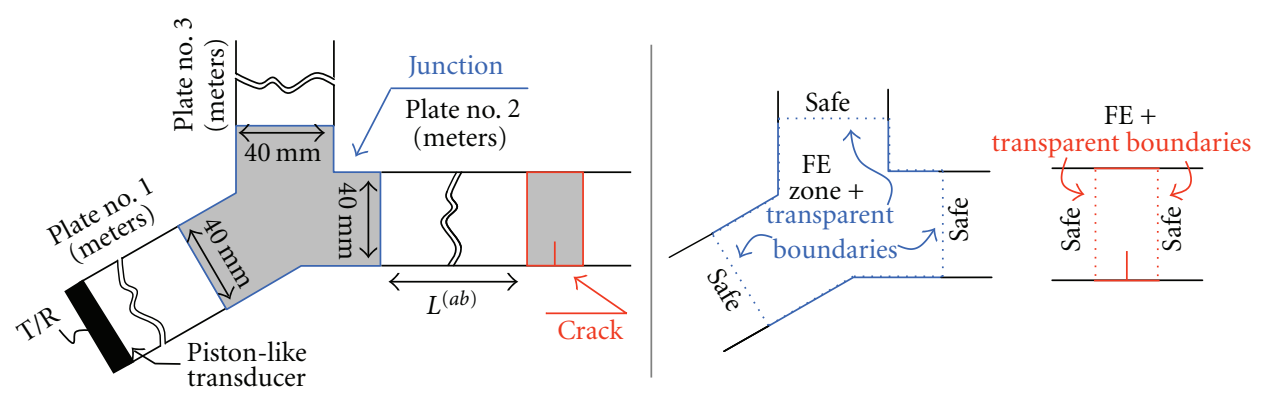

FIGURE 13: Left: configuration of testing of plate number 2 through a triple junction. Right: the two phenomena of scattering (junction, crack) are computed independently by FE; guided propagations are computed by SAFE.

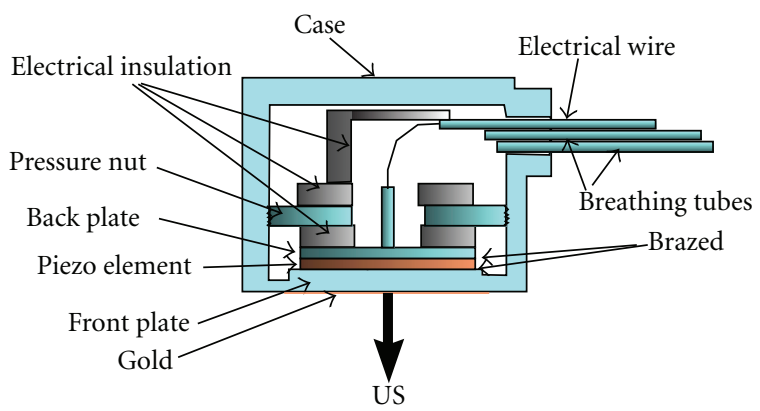

FIGURE 14: TUSHT general architecture.

$\left(200^{\circ} \mathrm{C}\right.$, short time operation), with better damping and time resolution.

Figure 15(b) shows the first AREVA prototype which has been manufactured and tested under sodium. It integrates a piezo electric ceramic. The associated results are quite satisfying. AREVA-NDE solutions were contracted to design and fabricate a transducer capable of performing NDE under liquid sodium. They developed an ultrasonic transducer called TUCSS, which is an acronym in French for "ultrasonic transducers for NDE under liquid sodium." This transducer employs a piezoelectric material for the generation of the ultrasonic pulse.

As shown in Figure 15, the outside packaging of this transducer is entirely metallic to prevent any risk of innerpart chemical degradation. Moreover, the front of the transducer is made of nickel, and the emissive surface has been subject to a mirror-polished surface treatment. This solution allowed to obtain an immediate acoustic coupling into liquid sodium at temperature as low as $110^{\circ} \mathrm{C}$.

For some applications, electro magnetic acoustic transducer (EMAT) is an interesting alternative to conventional piezoelectric transducers. The principle of an EMAT is to generate ultrasonic waves using the Lorentz force [11]. Eddy currents $J$ are induced into the inspected part thanks to a coil, while magnets generate the required magnetic field $B$. The interaction between these two fields creates the Lorentz force $F$, which produces ultrasonic waves, as shown in Figure 16. Reciprocally, the interaction of ultrasonic waves with a magnetic field induces currents in the receiving EMAT coil.
A monoelement EMAT probe has been developed for liquid sodium inspection at $200^{\circ} \mathrm{C}$. First experimental results are promising (see the first sampling in Figures 15(c) and 16). A full-phased array EMAT system is under development for sodium testing in 2012.

Further developments aim at using phased array transducers in order to get ultrasonic beam easy focusing and geometrical flexibility: this will be of a great importance when performing under sodium scanning for visualization purpose.

\subsubsection{Ultrasonic Transmission at $200^{\circ} \mathrm{C}$ Liquid Sodium/Trans-} ducer Interface. Good acoustic coupling between sodium and the active surface must be achieved when using an ultrasonic sensor immersed in sodium. During periodic examinations of the reactor at shutdown, the sodium temperature is about $180^{\circ} \mathrm{C}$. However, when a high-temperature ultrasonic transducer is immersed in sodium for the first time at this temperature, its acoustic coupling is not achieved [12].

The inprogress studies set out to assess the possibility of achieving good acoustic coupling of high-temperature ultrasonic transducers as soon as they are immersed in liquid sodium at reactor shutdown temperatures, without subjecting their active surface to vacuum gold plating. Gold plating the active surface of the sensor under vacuum makes it possible to achieve the acoustic coupling of the hightemperature ultrasonic transducer at the lowest temperatures at which liquid sodium can be used, that is, just above its melting point which is $98^{\circ} \mathrm{C}$ under atmosphere.

The attenuation generated by the gas present at the interface can be explained by considering:

(i) the relative amplitude of the acoustic impedances of steel, sodium, and of a gas (argon in the case of SFRs);

(ii) the expression of the energy transmission coefficient of acoustic waves between two elastic media.

In order to study the transmission of ultrasonic waves at a solid material-liquid sodium interface, we use the Liquidus sodium device which main components are a tight 400-liter containment (glove box under argon atmosphere) fitted with an airlock, and a 3-liter sodium pot whose temperature can be increased up to $450^{\circ} \mathrm{C}$. Two cylindrical metal bars made of 


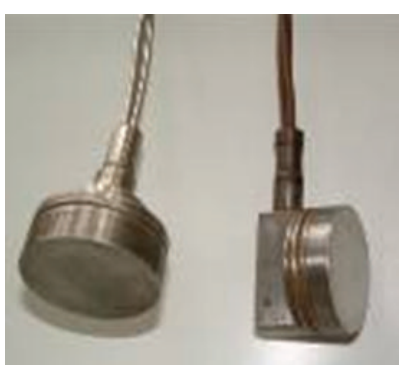

(a) TUSHT available CEA transducer (AISI 304L casing)

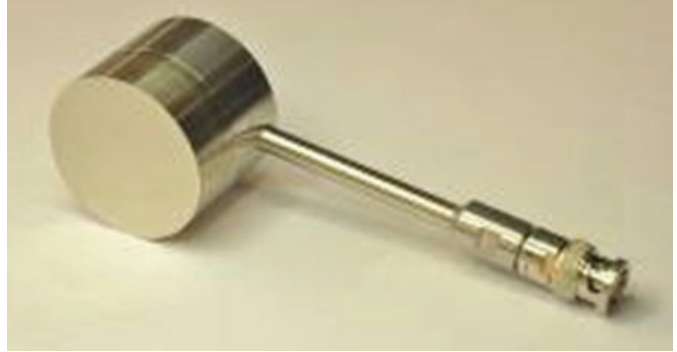

(b) TUCSS AREVA transducer (Nickel front face)

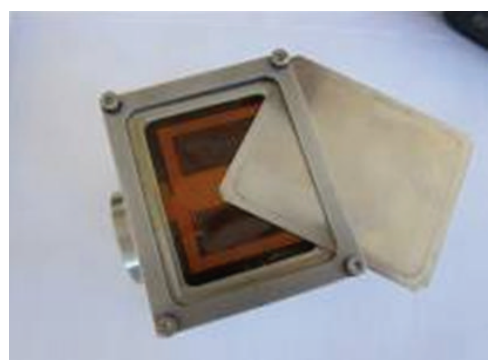

(c) EMAT CEA transducer (thin AISI 316L front face: here partially removed)

FIgURE 15: Available undersodium transducers.

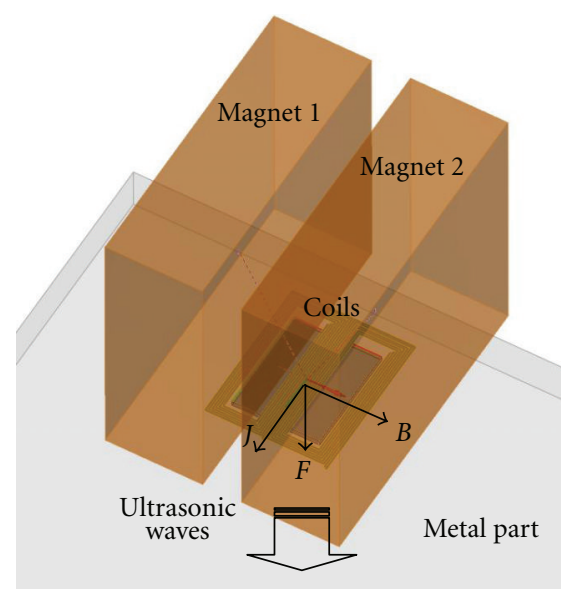

(a)

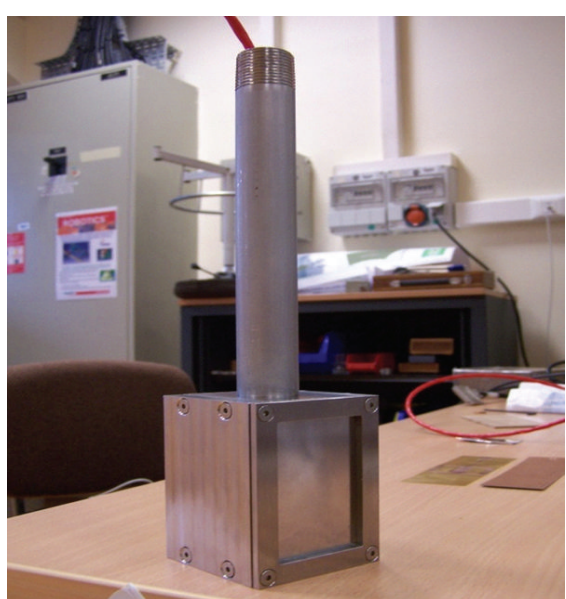

(b)

FIGURE 16: Principle of an EMAT and first insodium sampling.

316L steel, referred to as waveguides, are used for conveying the ultrasonic waves.

An ultrasonic transducer, employed as an emitter, is positioned at the free end of the left waveguide (Figure 17). An identical ultrasonic transducer, employed as a receiver, is positioned at the free end of the right waveguide.

The emitter transducer is excited by identical recurrent electrical pulses. The emitted wave propagates inside the left waveguide, passes through the left waveguide,sodium interface and then through the sodium/right waveguide, propagates inside the right waveguide and finally excites the receiver transducer.

During the tests, the variations of the electrical signal generated by the receiver transducer are recorded. Based on the assumption that transducer-waveguide couplings remain identical over time, the electrical signal generated by the receiver transducer provides information on the transmission of ultrasounds at sodium-waveguide interfaces.

The interpretation of these results is based on the assumption that acoustic coupling is improved due to a decrease in the gas surface fraction at the interface. Inert gases, and particularly argon, are used as cover gases for liquid sodium in fast reactors. Their solubility in sodium increases exponentially with temperature. If the temperature of sodium is increased, part of the gas present in the crevices of the rough surface dissolves and diffuses to the sodium [13].

Furthermore, in addition to increasing solubility, a temperature rise also increases the diffusion coefficient (or diffusivity) of argon in sodium [13]. This phenomenon accelerates the dissolution of the gas contained inside the crevices and as a result accelerates the decrease of the gas surface fraction at the interface.

Lastly, another temperature-related effect is observed. This effect has the most significant impact on the gas surface fraction and consists in the variation of the contact angle according to temperature.

The combination of these three effects leads to an interpretation based on two main observations. The first observation is that a good acoustic coupling is achieved at the same temperature at which the wetting process is observed. The second observation is that the increase rate of transmitted energy increases with temperature. In this interpretation, wetting consists in the dissolution of gas pockets.

4.3.3. Liquid Sodium Ultrasonic Telemetry Experimental Tests. In order to qualify CIVA software (see Section 4.2), an 


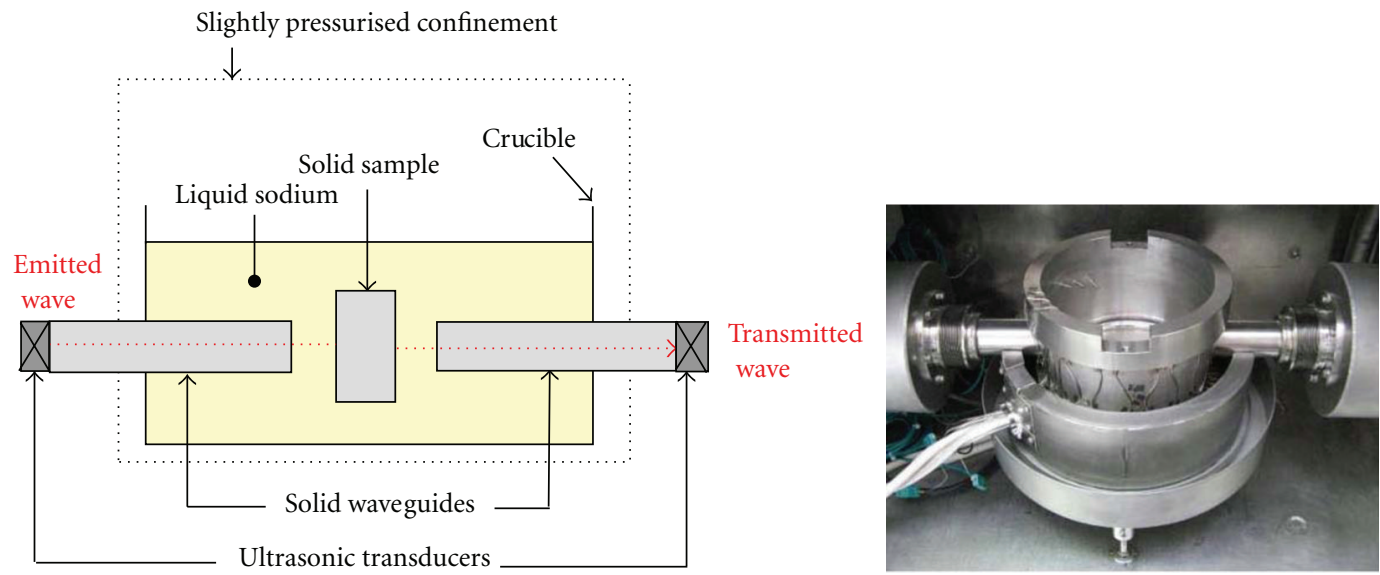

FIgURE 17: Schematic view of the acoustic path of Liquidus device and view of the facility (with 210 mm diameter sodium pot).

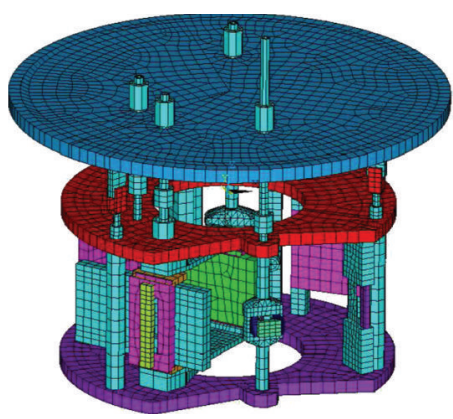

(a)

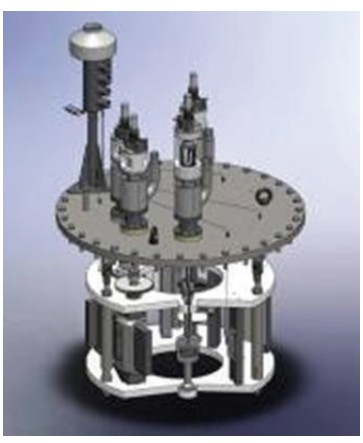

(b)

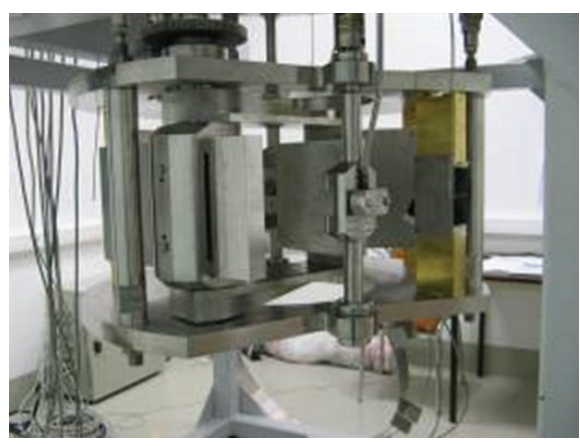

(c)

FIGURE 18: multireflector mockup ( $1 \mathrm{~m}$ diameter) devoted to in sodium ultrasonic telemetry study.

experimental mockup "multireflector" was specified for the study of ultrasonic diffractions and reflections in liquid sodium and was successfully tested in 2010 [14]. It included a rotating high-temperature ultrasonic transducer TUSHT, a fixed target, rotating targets, and thermocouples (Figure 18). In order to reach the metrological objective, in air initial calibration at room temperature of all components was performed and led to a global uncertainty equal to $\pm 0,02 \mathrm{~mm}$ $(20 \mu \mathrm{m})$ for their location and to $\pm 0,02^{\circ}$ for their angular position.

After under-water commissioning tests, the mockup was used in a $1 \mathrm{~m}$ diameter pot inisothermal $200^{\circ} \mathrm{C}$ static sodium conditions: test parameters were TUSHT frequency and 6 target positions. Then, global uncertainty on ultrasonic distance measurement could be checked and is better than $100 \mu \mathrm{m}$.

4.3.4. Liquid Sodium Ultrasonic Visualization. Extending telemetric measurement leads to the possibility of ultrasonic under sodium "vision," also called, "visualization" able to deal with different applications:

(i) surface mapping (imaging) of submerged structures/components; (ii) integrity inspection of structure/component surfaces (including the detection and sizing of opened cracks);

(iii) determination/confirmation of robotic system positioning;

(iv) fuel assembly identification;

(v) detection, localization and sizing of immersed objects (including migrating bodies).

$\mathrm{R} \& \mathrm{D}$ program is beginning in 2012 and intends to compare all the identified ultrasonic technologies available for surface telemetry options: orthogonal antenna (former CEA IMARSOD project: see Figure 19 [15]), conventional or innovative SAFT (synthetic aperture focusing technique), 2D matrix with Full Matrix Capture for example (Figure 19).

Depending on the visualization system chosen, in water tests will be performed in CEA VISIO facility (Figure 20). Surface examination under sodium conditions seems possible and could be used also for:

(i) surface metrology: measuring shape defects, detecting, and measuring through crack openings; 


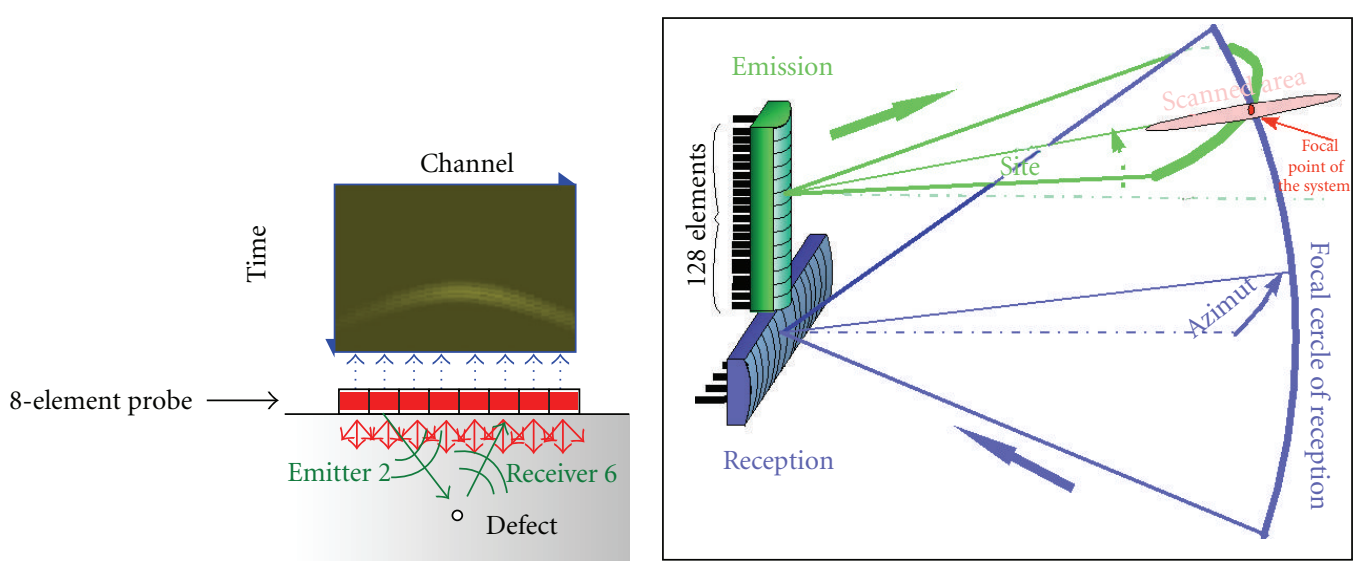

(a) full matrix capture (FMC)-Phased array technique

(b) IMARSOD antenna-phased array technique

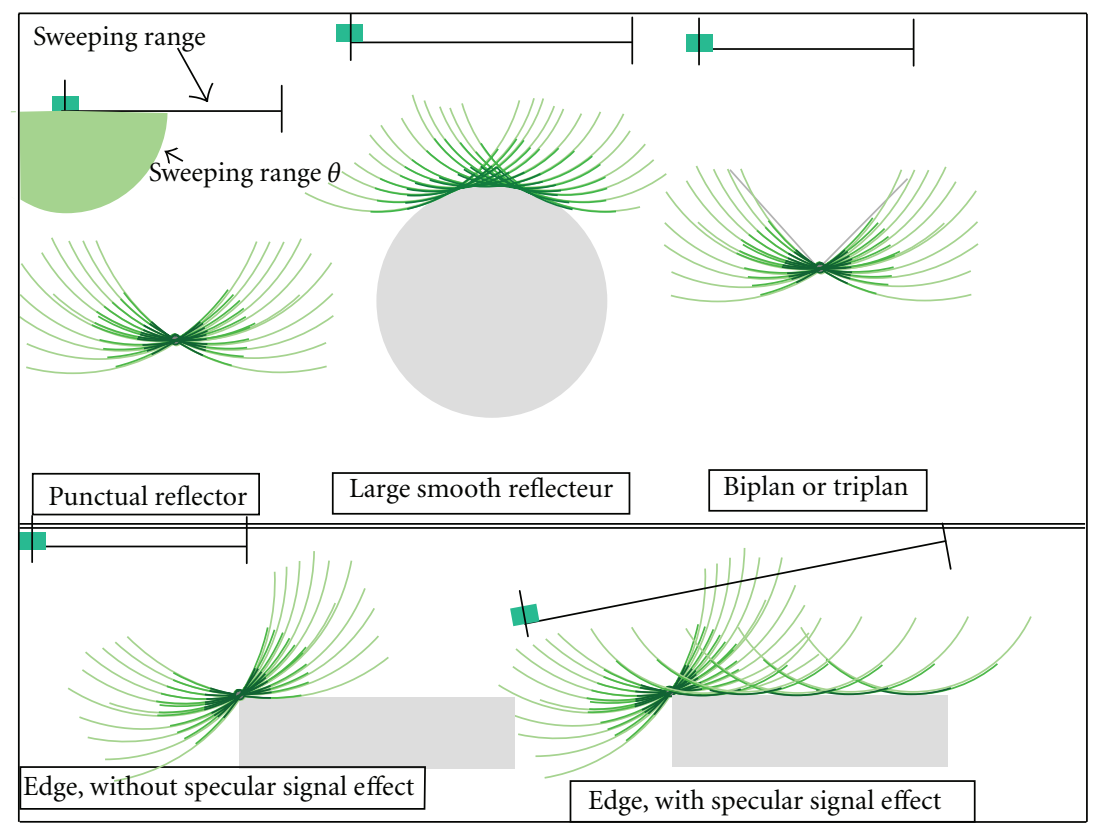

(c) synthetic aperture focusing technique. (SAFT)

Figure 19: Ultrasonic visualization techniques (examples).

(ii) remote operations under sodium viewing: detecting and measuring positions and dimensions of migrating bodies, detecting the absence of mechanical parts, and helping to position a robot.

The different ultrasonic techniques are considered to be more or less adapted to specific cases where ultrasonic echoes can come from diffraction on edges, reflection on flat surfaces, and retrodiffusion due to surface roughness. Specification for vision performance can be extended from optical case (capability of detection, resolution, and accuracy): a first step can be considered to assume ASME rules which specify a $800 \mu \mathrm{m}$ capability of detection. Ultrasonic techniques imply rotating and translating scanning which can be realized with mechanical or electronic phased array systems. Directivity of ultrasonic sensors will have a great importance, depending on acoustic technique to be used.
Underwater tests will first be performed and then sodium tests will be carried out when ultrasonic corresponding sensors will become available.

4.4. Ultrasonic Control without Sodium-Immersed Sensors. The harsh environmental constraints inside the main vessel require developing high-temperature transducers conditioned for sodium. But conditioning greatly affects the performances of the transducer such as the SNR ratio. It is why ultrasonic probes positioned outside the reactor along the main vessel wall can be used to perform telemetry measurement without requiring previous sodium conditioning.

Such examination seems to be possible for some internal peripheral structures: it could be facilitated by an external mean, displayed from the inter-vessel gap $[16,17]$.

As said before, such an ultrasonic method is being investigated for the two main purposes (see Figure 4): 


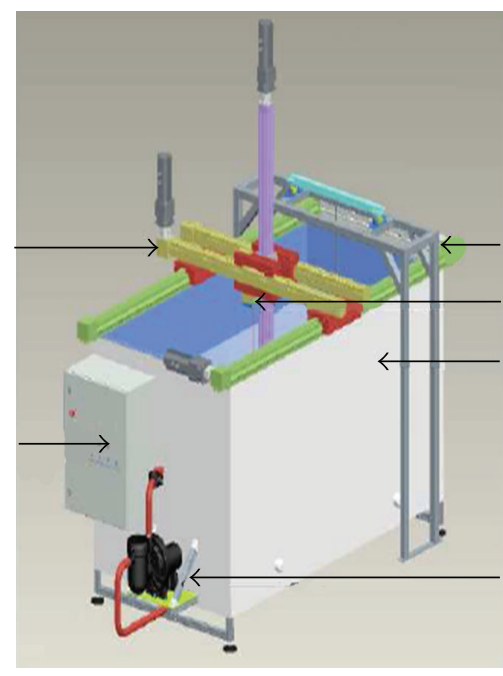

(a)

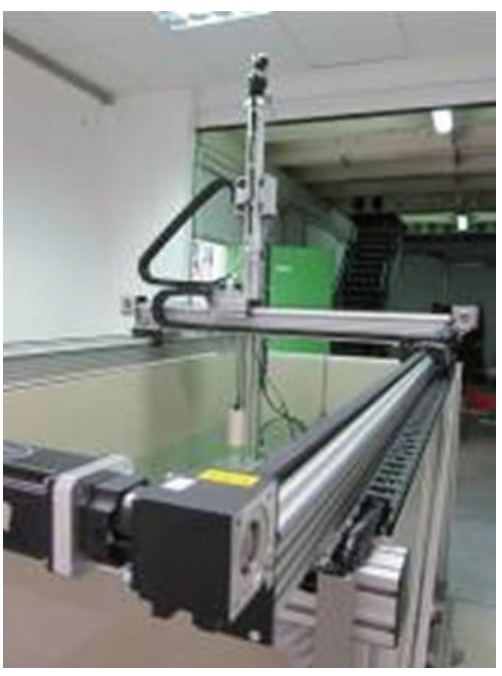

(b)

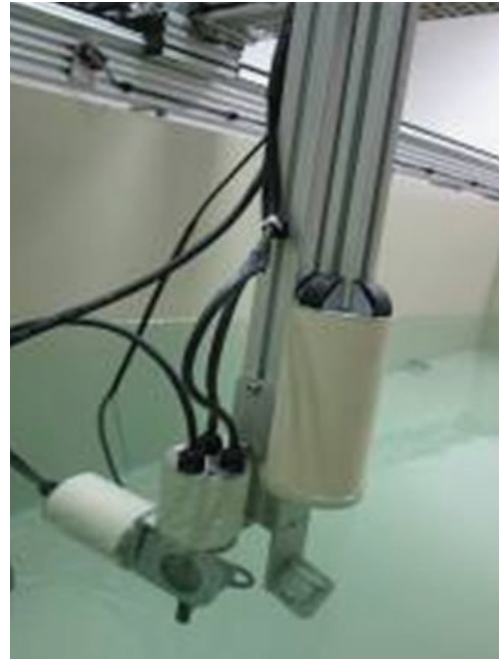

(c)

FIgURE 20: VISIO water facility ( $2 \mathrm{~m}$ long, $1 \mathrm{~m}$ large, and $1 \mathrm{~m}$ high) devoted to ultrasonic visualization study.

(i) telemetric measurements in order to check the immersed structure positioning and thickness;

(ii) volumetric NDE of the core support peripheral welded junctions in order to verify the absence of cracks.

Transfer matrix method is used in order to simulate the ultrasonic transmission through several immersed plates. The need to propagate the maximum energy possible through bounded media orientated the study towards Lamb waves. These waves are often employed for single plates or solid layers but are rarely used for liquid/solid pile. The set of alternating steel plates and layers of liquid sodium can be linked to a multilayer system. A common method for studying the propagation of waves in a theoretical layered medium is the method of transfer matrices. The idea is to build the propagation matrix for a stack of a known number of parallel layers by extending the solution from one layer to the next and satisfying the continuity conditions at interfaces. The principle is $[18,19]$

(i) each layer of the system is associated with a transfer matrix, connecting the velocities and stresses at the "outer" interface (downstream) in terms of velocities and stresses at the "inner" interface (upstream);

(ii) the transfer matrix of the complete system is obtained by multiplying the transfer matrices of the constituent layers.

Therefore, the matrix can be used to determine the transmission and reflection coefficients of the system under the assumption of an incident plane wave. This calculation takes into account the interferences due to multiple reflections and possible resonances.

The transmission coefficients are calculated and represented by varying angle of incidence and frequency. The dynamic range varies between 0 (dark blue) and 1 (red), with large areas where the coefficient is about 0.1 (blue). It can be observed that the generated Lamb modes in a system with two plates (see maxima of Figure 21) are exactly the same as all Lamb modes generated in each individual plate. There is no mixed mode generated by the systems of $n$ plates. Only the sum of elementary modes generated by each plate is present.

In order to qualify this method, tests in water have begun investigating various influential parameters (frequency, pulse mode or wave trains, sheet thickness, spacing, number of plates and parallelism), meanwhile simulation codes, that is, CIVA (see Section 4.2), must be developed and/or adapted to evaluate and compare the considered reactor designs from the method applicability point of view. Insodium testing will follow. Experimental tests will be conducted in water and then in sodium.

The experiments (Figure 22) are carried out in immersion using a broadband $\mathrm{E} / \mathrm{R}$ transducer with a central frequency of $1 \mathrm{MHz}$. Two incidence angles $\left(18^{\circ}\right.$ and $\left.35^{\circ}\right)$ are used to generate the Lamb modes A0 and S0 in the last plate. The signals are sent back by an artificial defect (notch) located in the last plate and recorded. The maximum amplitude of the signal is represented as a relative versus the distance from the notch.

The results (Figure 23) show that it is possible to generate a Lamb wave in a plate hidden behind two others, except in the case of different thicknesses and S0 mode. These screens can be of the same thickness or not. The amplitude of this wave consistently decreases with the distance from the reflector (notch).

It can be observed that the relative decrease in the case of three plates and A0 mode is lower than in the case of one and two plates.

It can be seen that an artificial defect can be detected in a plate located behind one or two screens by generating Lamb waves in the last plate. The measured attenuation on this scale is compatible with industrial NDE conditions. Thus, this inspection technique shows promising potential. 


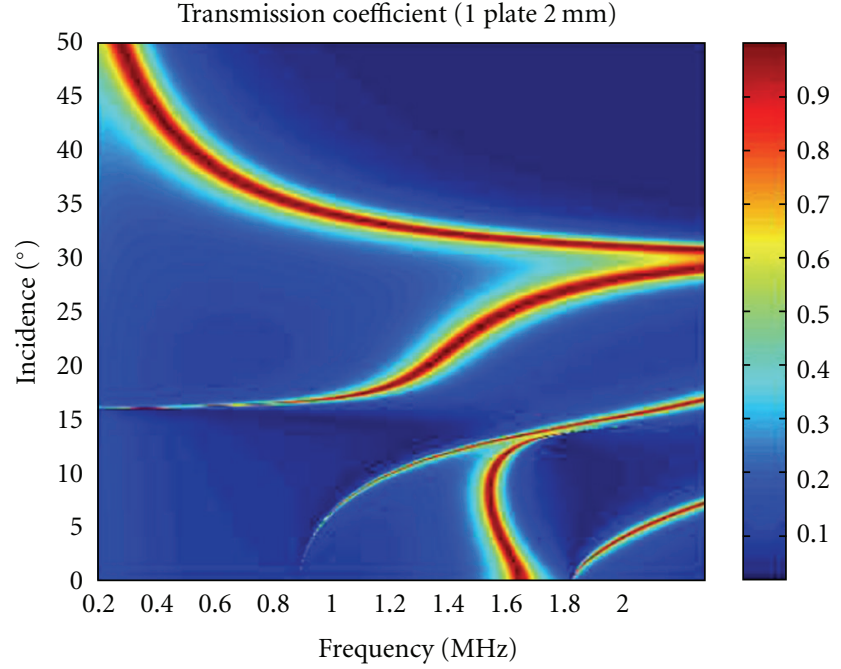

(a)

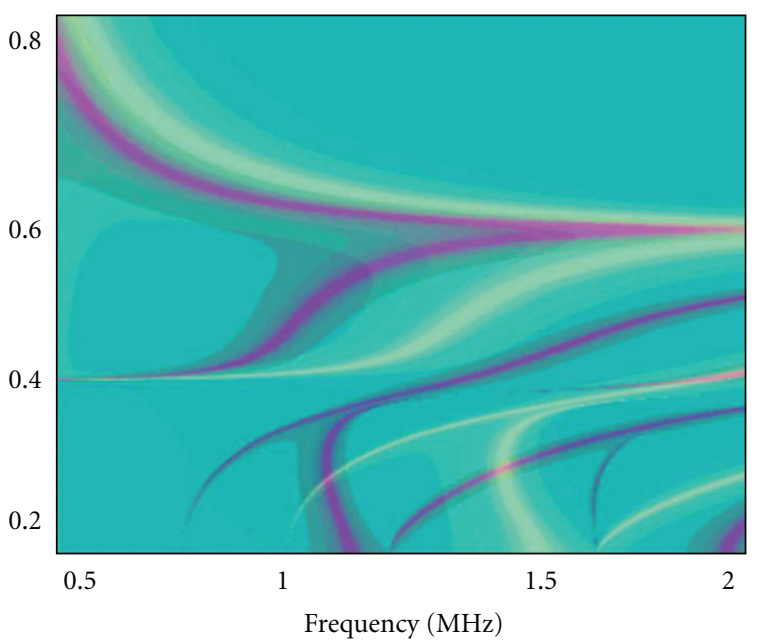

(b)

Transmission coefficient

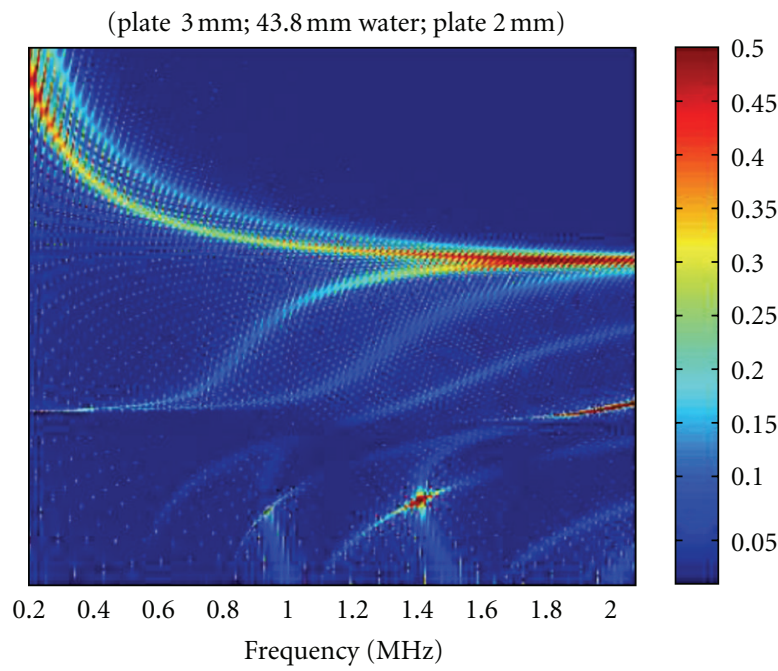

(c)

Figure 21: Transmission coefficients calculated for different systems: (a) a single $2 \mathrm{~mm}$ thick plate: Lamb wave modes appear very clearly, (b) graphical superposition for a single $2 \mathrm{~mm}$ thick plate and for a $3 \mathrm{~mm}$ thick plate: each plate has its own Lamb wave modes, (c) two plates ( $2 \mathrm{~mm}$ thick and $3 \mathrm{~mm}$ thick): there is a global Lamb wave mode filtering.

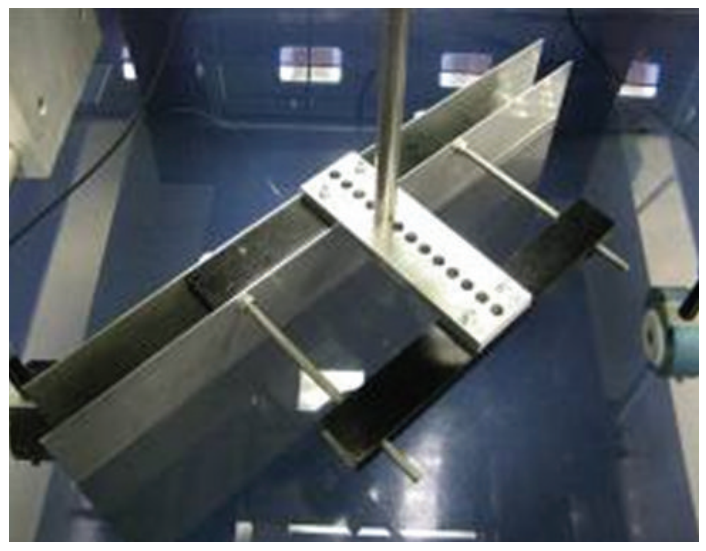

FIGURE 22: Inwater experimental mockup (300 mm square plates).
Further study will focus on identifying the plate containing the defect, and on the location of the crack.

Larger-scale experiments are being performed in water (Figure 24): 3 thick plates (20 and $30 \mathrm{~mm}$ ) are immersed in water and submitted to $100 \mathrm{kHz}$ acoustic waves in order to develop telemetry (distance between plates and plate thickness measurement) and volumetric NDE (detection and sizing of volumetric defects in the plates) applications. Some artificial defects have been machined in the plates: slits, holes, and grooves.

Then, qualification phase will be performed with tests in liquid sodium conditions.

4.5. Ultrasonic Surface Wave Performing. As evoked in the global design paragraph, several inner structures may 


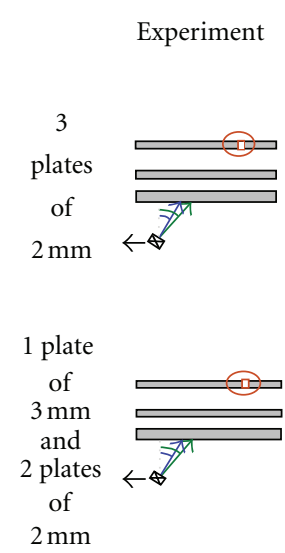

System transmission coefficients
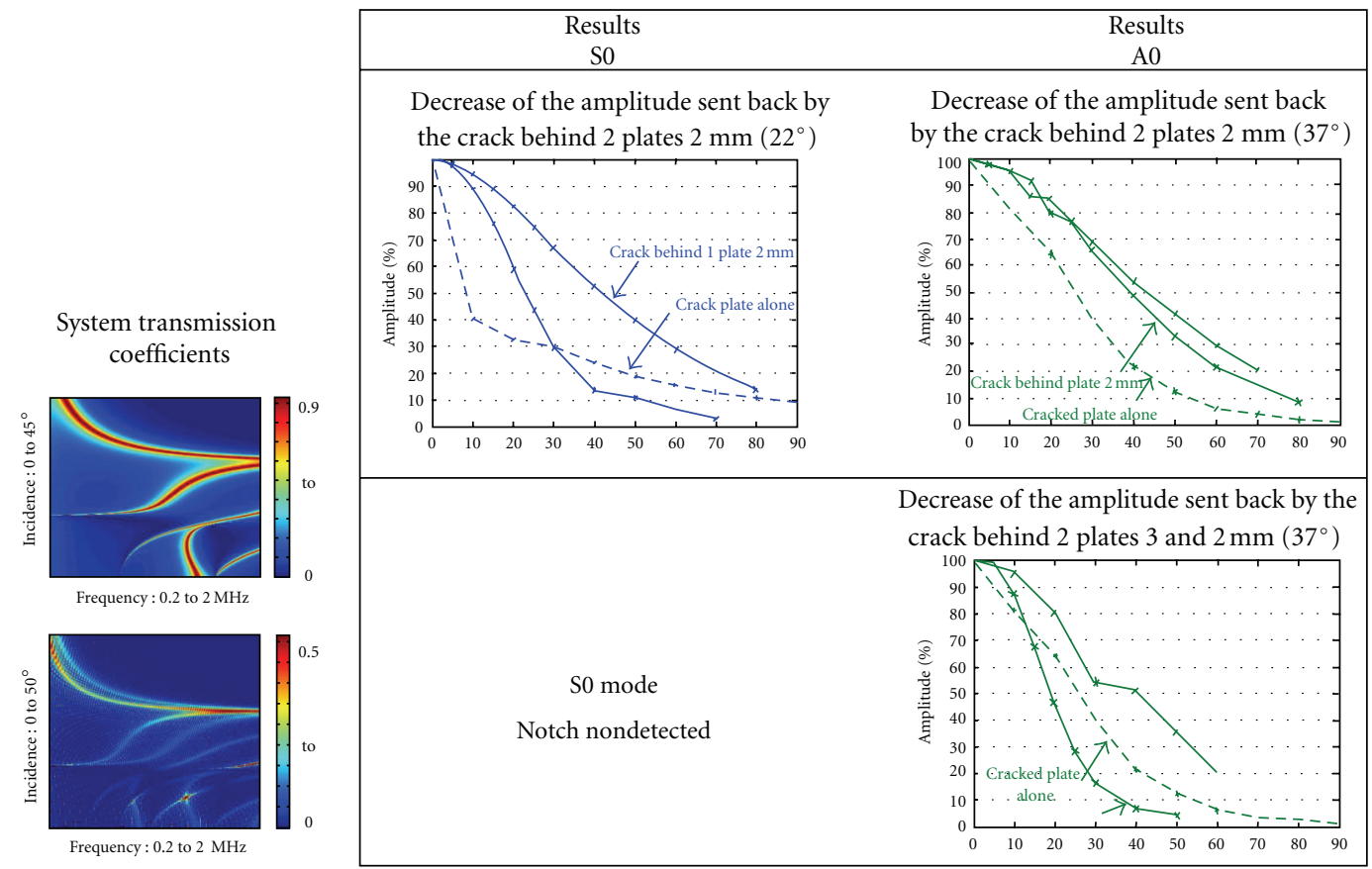

FIGURE 23: The results of three plates (solid lines) are compared in terms of the relative amplitude with the results of one and two plates (dashed lines).

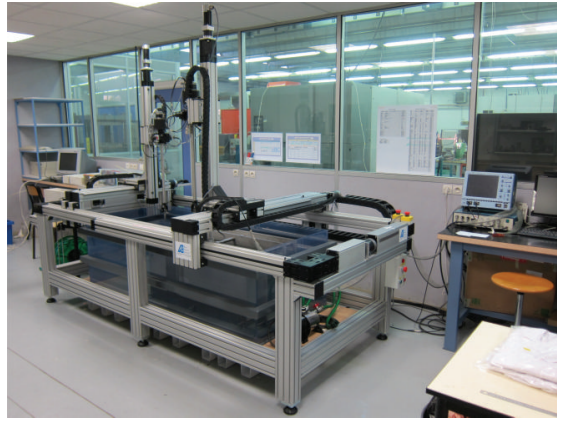

(a)

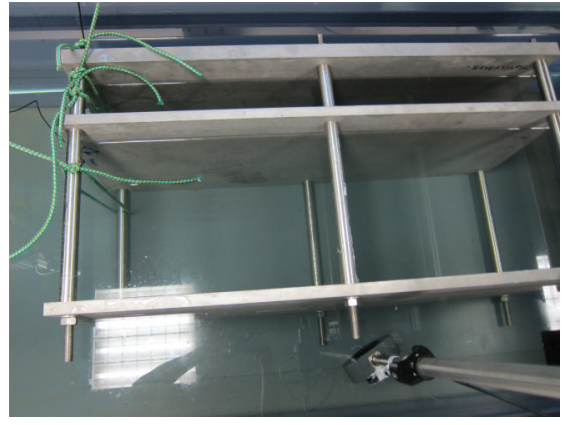

(b)

Figure 24: Scale 1 in water tests with a 3-plate (1.5 m long and $1 \mathrm{~m}$ large) mockup.

undergo some thermal striping, especially the ACS lower zone. To be able to detect the resulting superficial cracks, a specific $R \& D$ program has been implemented.

The first tests on a representative mockup in air have shown that propagating US surface waves give some good results, detecting the artificial cracks positioned onto the mockup, as shown in Figure 25. The sensors used were monoelement with variable angle focus and phased array.

Next testing will analyze the method applicability for longer detection distance and immersed structure with a liquid free level.

4.6. Sodium Environment Repair. For the nonremovable components, repair operations will be performed in a gas environment: either in the upper dry zones of the reactor cover gas plenum, or in a gas-tight volume, if the faulty zone is located under the sodium free level. In that last case, the gas-tight system will have to contain the inspection and repair tools and protect them from the surrounding liquid sodium (Figure 26). Two concepts will be investigated: one in contact with the structures, having a seal formed by two flexible lips, and the other is a system that prevents liquid sodium penetration by using a gas-pulsed screen, supplemented by a scraper seal. Specific seal material is being tested in $200^{\circ} \mathrm{C}$ liquid sodium: conventional PWR silicone material is being tested for sodium qualification (Figure 26).

Concerning the repair tools, on the one hand, the laser method has been selected for future SFRs because it has the advantage of not generating any stress on the tool, and because it is suitable for the various applications: indeed, the repair scenario for insodium structures should be first removal of sodium (after bulk sodium draining), then machining and finally welding. On the other hand, the 


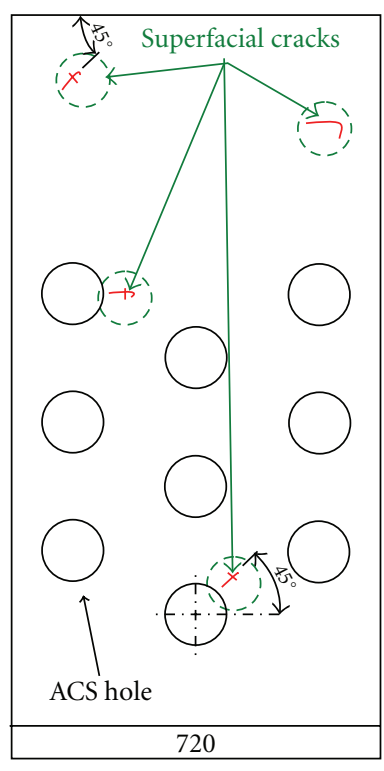

ACS mock up

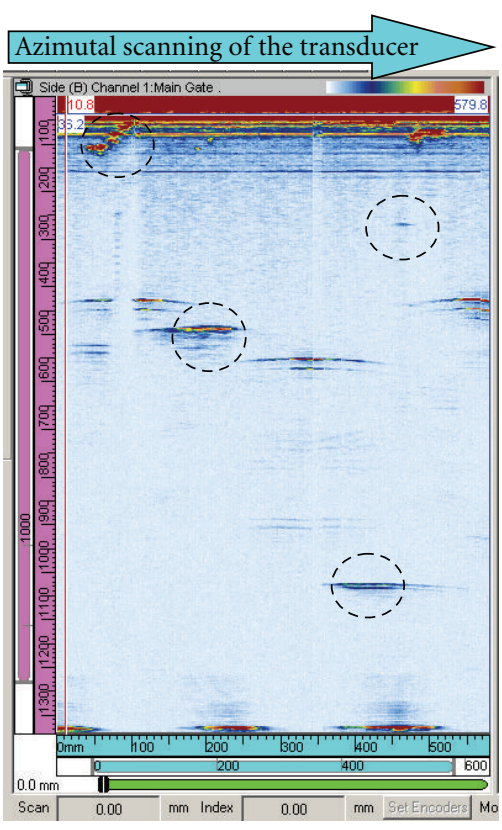

B-scan (screen copy)

FIGURE 25: Surface ultrasonic controls of thermal striping cracks. Echoes are due to geometrical boundaries (holes) and to superficial cracks.

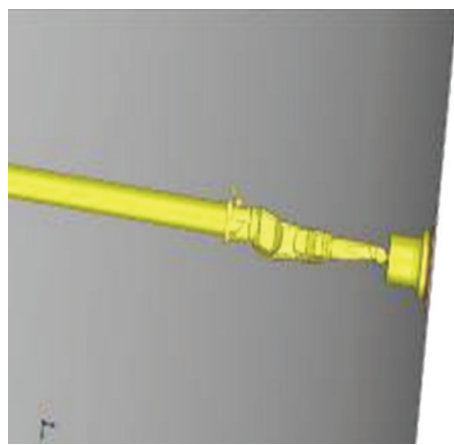

(a) insodium robot concept: articulated arm with a bell

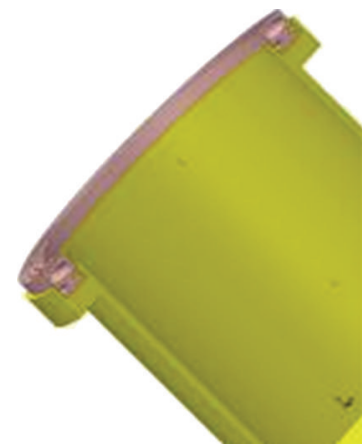

(b) gas-tight bell design for Repair tools

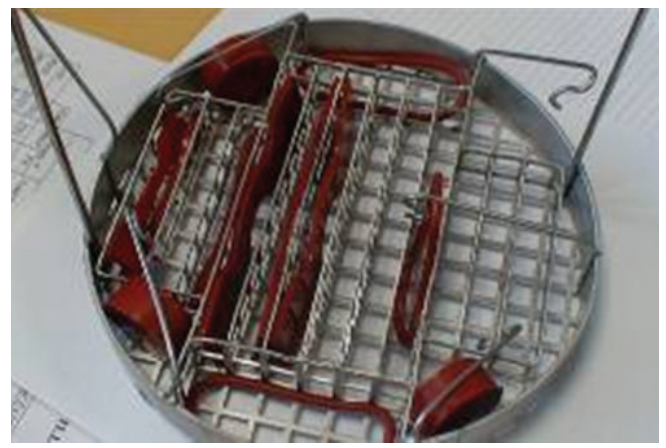

(c) silicone samples before sodium testing

FIgURE 26: Tools for insodium intervention.

conventional tools (brush or gas blower for sodium removal, milling machine for machining, and TIG for welding, whose feasibility was demonstrated in the 1990s) are still considered as backup solution. Qualification tests have to be performed with the help of simulation of welding process (Figure 27).

In any case, the waste produced (smoke and aerosols; dust and chips) will have to be managed.

Sodium complete or partial draining induces a caustic corrosion risk which has to be considered carefully as it can produce fast cracking in stainless steel material of SFR structures: this is due to possible chemical reaction of sodium traces with oxygen or moisture in case of air ingress. If produced sodium hydroxide is wetted and if structure stress is high enough, stress corrosion cracking is likely to occur, depending on the temperature range [20]. That can happen when draining sodium but also during refilling it.
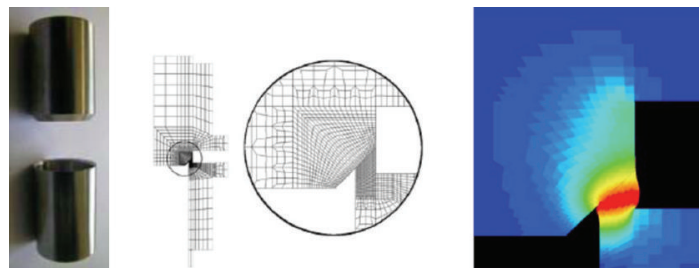

FIGURE 27: Simulation of electrothermic welding process for small samples (some mm thickness).

Even if associated intervention procedures are available, investment protection needs to study the case of complete draining of primary sodium (out of the main vessel), for both scenarios corresponding to shutdown $200^{\circ} \mathrm{C}$ and room 


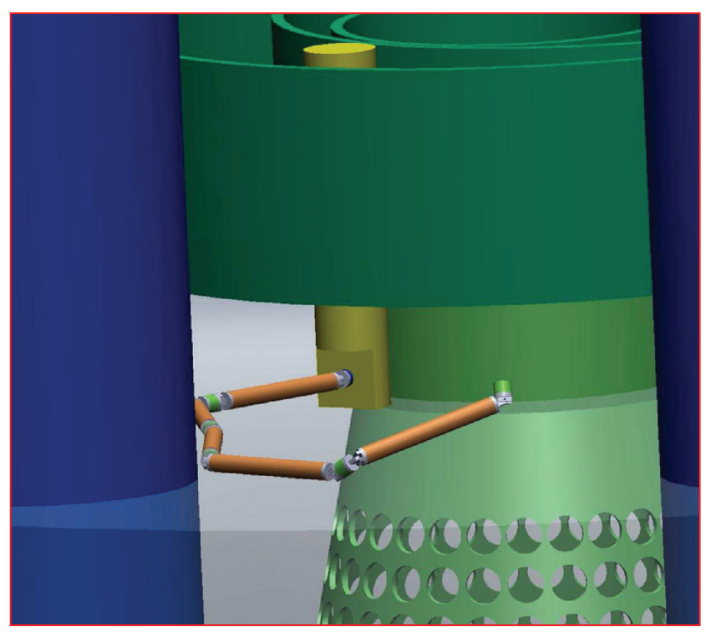

FIGURE 28: Insodium robotics for inspection/repair (CAD simulation example): articulated arm with NDE inspection too.

temperature conditions. Simulation of air ingress aerolics and chemical reaction with sodium traces will be performed.

4.7. Robotics for Insodium Examination and Repair. In pile deployments for examination or repair require some robotic carriers. These carriers have to be suitable for sodium environment: either in the cover gas plenum or in gas after sodium draining, or even under liquid sodium.

Few insodium carriers have been used in the past: it is why a specific R\&D program has been initiated with the objective to have a prototype of robotic carrier for insodium testing by 2014 .

This $\mathrm{R} \& \mathrm{D}$ program is parted in 10 axes:

(i) detailed definition for the SFR carrier needs (internal structure access, and possible defects to be detected/ repaired);

(ii) carrier architecture definition and specifications (depending on inspection and repair scenarios);

(iii) carrier organ in sodium tightness;

(iv) carrier material compatibility with sodium;

(v) temperature resistance $\left(200^{\circ} \mathrm{C}\right)$;

(vi) irradiation resistance (depending on location of main vessel);

(vii) intervention gas-tight bell for operation under liquid sodium;

(viii) carrier positioning control on liquid sodium;

(ix) development, validation and qualification of technological solutions, then of carrier fleet;

(x) worldwide benchmark regarding the previous axes.

The research work along these 10 axes must allow choosing between the adaptation of manufactured robots, in order to benefit from the reliability and the industrial experience, and the designing of specific robots compatible with the surrounding conditions (drive systems, coding, reduction, tightness, and data exchange).

In addition to this approach, the continuation of accessibility studies based on reactor design studies will help to optimize the accessible volume to a robot (Figure 28).

\section{Conclusion}

On the basis of available feedback and the new safety requirements, the ISI\&R for next SFRs has been identified as a major task. The French $\mathrm{R} \& \mathrm{D}$ program launched in 2007 for periodic examination and repair improvement is based on several aspects: ensuring a strong connection between the reactor designers and inspection specialists, and developing tools and techniques applicable in a sodium environment (inspection at $200^{\circ} \mathrm{C}$ ). The key milestones (and main available results) of this ambitious $\mathrm{R} \& \mathrm{D}$ program are:

(i) validation for ultrasonic simulation (achieved for isothermal sodium conditions);

(ii) validation of ultrasonic transducers (US telemetry and NDE sensors) under sodium conditions at $200^{\circ} \mathrm{C}$ (procedures for manufacturing of available CEA designed TUSHT have been validated);

(iii) definition of key components of the robotic equipment for operation in sodium at $200^{\circ} \mathrm{C}$ (in sodium seals and thermal isolation are being qualified);

(iv) validation of laser repair processes and techniques: sodium sweeping, structure machining, and welding (simulating zinc test are being performed).

In the frame of this R\&D activity, feasibility and qualification tests are performed, simulation of techniques (ultrasonic inspection and laser repair) are developed, inspection and repair tools are designed, manufactured, and tested.

ASTRID prototype project orientates these actions and will get large benefits from them.

\section{Acknowledgment}

The authors would like to thank the colleagues who provided information or checked certain sections of this paper.

\section{References}

[1] F. Jadot, F. Baqué, G. de Dinechin, J. P. Jeannot, J. M. Augem, and J. Sibilo, "ASTRID Sodium cooled Prototype : program for improving in service inspection and repair," in Proceedings of the 2nd International Conference on Advancements in Nuclear Instrumentation, Measurement Methods and their Applications (ANIMMA '11), Ghent, Belgium, June 2011, Paper 3-37.

[2] F. Baqué, G. Rodriguez, N. Jardin, J. M. Carpreau, J. M. Augem, and J. Sibilo, "Generation IV nuclear reactors-R\&D program to improve sodium-cooled systems inspection," in Proceedings of the 1st International Conference on Advancements in Nuclear Instrumentation Measurement Methods and their Applications (ANIMMA '09), Marseille, France, June 2009, Paper 77.

[3] J. F. Sauvage, Phénix 30 Years of History: The Heart of a Reactor, CEA, Paris, France, 2004. 
[4] J. Sibilo, E. Breuil, F. Baqué, and J. M. Augem, "Generation IV nuclear reactors: strategy and challenges of R\&D program for improving inspection and repair of sodium cooled systems," in Proceedings of the International Congress on Advances in Nuclear Power Plants (ICAPP '10), San Diego, Calif, USA, June 2010, Paper 8096.

[5] F. Reverdy, F. Baqué, B. Lu, K. Jezzine, V. Dorval, and J. M. Augem, "Simulation of ultrasonic inspection for sodium cooled reactors using CIVA," in Proceedings of the 2nd International Conference on Advancements in Nuclear Instrumentation, Measurement Methods and their Applications (ANIMMA '11), Ghent, Belgium, June 2011.

[6] M. Darmon, N. Leymarie, S. Chatillon, and S. Mahaut, "Modelling of scattering of ultrasounds by flaws for NDT," in Ultrasonic Wave Propagation in Non Homogeneous Media, vol. 128 of Springer Proceedings in Physics, pp. 61-71, Springer, Berlin, Germany, 2009.

[7] R. H. Kraichnan, "Diffusion by a random velocity field," Physics of Fluids, vol. 13, no. 1, pp. 22-31, 1970.

[8] K. Jezzine and A. Lhemery, "Simulation of guided wave inspection based on the reciprocity principle and the semi-analytical finite element method," in Proceedings of the AIP Conference Proceedings, D. O. Thompson and D. E. Chimenti, Eds., vol. 894 of In Review of Progress in QNDE, 26A, pp. 39-46, American Institute of Physics, Melville, NY, USA, 2007.

[9] A. Lhemery, V. Baronian, K. Jezzine, and A.-S. BonnetBenDhia, "Simulation of inspections of elastic waveguides of arbitrary section containing arbitrary local discontinuities or defects," in Proceedings of the AIP Conference Proceedings, D. O. Thompson and D. E. Chimenti, Eds., vol. 1211 of In Review of Progress in QNDE, 29A, pp. 145-152, American Institute of Physics, Melville, NY, USA, 2010.

[10] C. Lhuillier, O. Descombin, F. Baqué, B. Marchand, J. F. Saillant, and J.M. Augem, "In sodium tests of ultrasonic transducers," in Proceedings of the International Conference on Advancements in Nuclear Instrumentation, Measurement Methods and Their Applications, Ghent, Belgium, June 2011, Paper 3-06.

[11] H. M. Frost, "Electromagnetic-ultrasound transducers: principles, practice, and applications," Physical Acoustics, vol. 14, pp. 179-275, 1979.

[12] O. Descombin, K. Paumel, J. Moysan, G. Corneloup, and J.M. Augem, "Acoustic coupling of ultrasonic transducers for in-service inspection of sodium fast reactors," in Proceedings of the International Conference on Advancements in Nuclear Instrumentation, Measurement Methods and Their Applications (ANIMMA '09), Marseille, France, June 2009, Paper 78.

[13] P. K. Holland and R. H. S. Winterton, "Nucleation of sodium boiling from surface cavities containing gas," International Journal of Heat and Mass Transfer, vol. 16, no. 7, pp. 14531458, 1973.

[14] G. Gobillot, F. Baqué, C. Lhuillier et al., "Ultrasonic techniques for improving inspection of sodium-cooled systems," in Proceedings of the International Conference on Advancements in Nuclear Instrumentation, Measurement Methods and Their Applications (ANIMMA '09), Marseille, France, June 2009, Paper 80.

[15] C. Imbert, J. L. Berton, and G. Gimenez, "Realization of ultrasonic images of immersed metallic structures using a digital beamforming system. Experimental study," in Proceedings of the 5th International Conference on Nuclear Engineering (ICONE5 '97), p. 411, May 1997.

[16] G. Corneloup, M. A. Ploix, J. F. Chaix, I. Lillamand, and F. Baqué, "Potential of ultrasounds for NDT of a structure located behind parallel immersed plates," in Proceedings of the AIP Conference Proceedings (QNDE '10), San Diego, Calif, USA, July 2010.

[17] F. Baqué, K. Paumel, G. Corneloup, M. A. Ploix, and J. M. Augem, "Non destructive examination of immersed structures within liquid sodium," in Proceedings of the International Conference on Advancements in Nuclear Instrumentation, Measurement Methods and Their Applications (ANIMMA '11), Ghent, Belgium, June 2011, Paper 3-07.

[18] A. H. Nayfeh, "Elastic wave propagation in fluid-loaded multiaxial anisotropic media," Journal of the Acoustical Society of America, vol. 89, no. 2, pp. 542-549, 1991.

[19] B. Hosten and M. Castaings, "Transfer matrix of multilayered absorbing and anisotropic media. Measurements and simulations of ultrasonic wave propagation through composite materials," Journal of the Acoustical Society of America, vol. 94, no. 3, pp. 1488-1495, 1993.

[20] L. Champeix, P. Baqué, and C. Chairat, "Stress corrosion on austenitic stainless steels components after sodium draining," in Proceedings of the 2nd International Conference on Liquid Metal Technology in Energy Production, pp. 49-55, Richland, Washington, DC, USA, April 1980. 

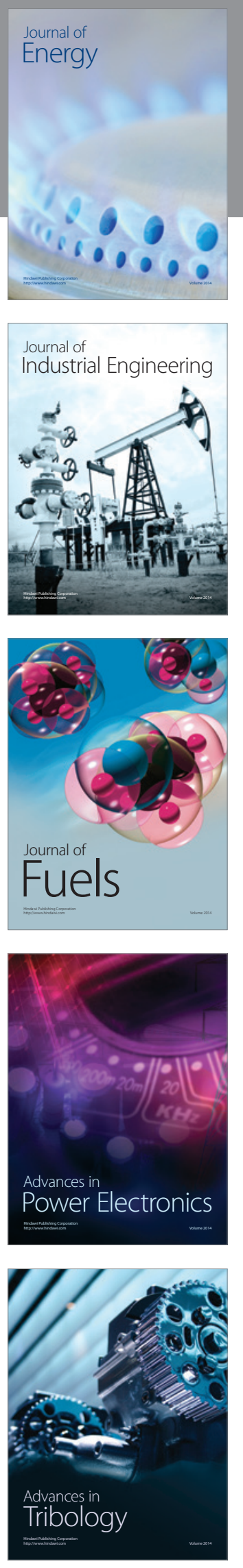
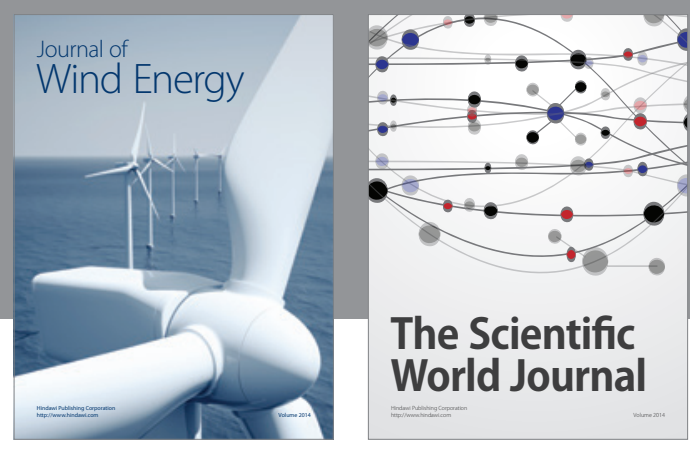

The Scientific World Journal

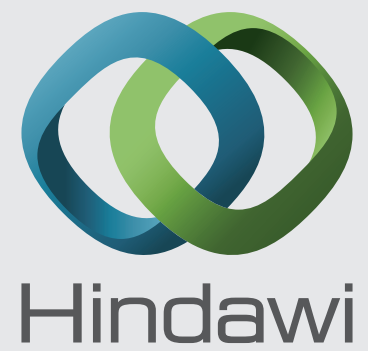

Submit your manuscripts at http://www.hindawi.com
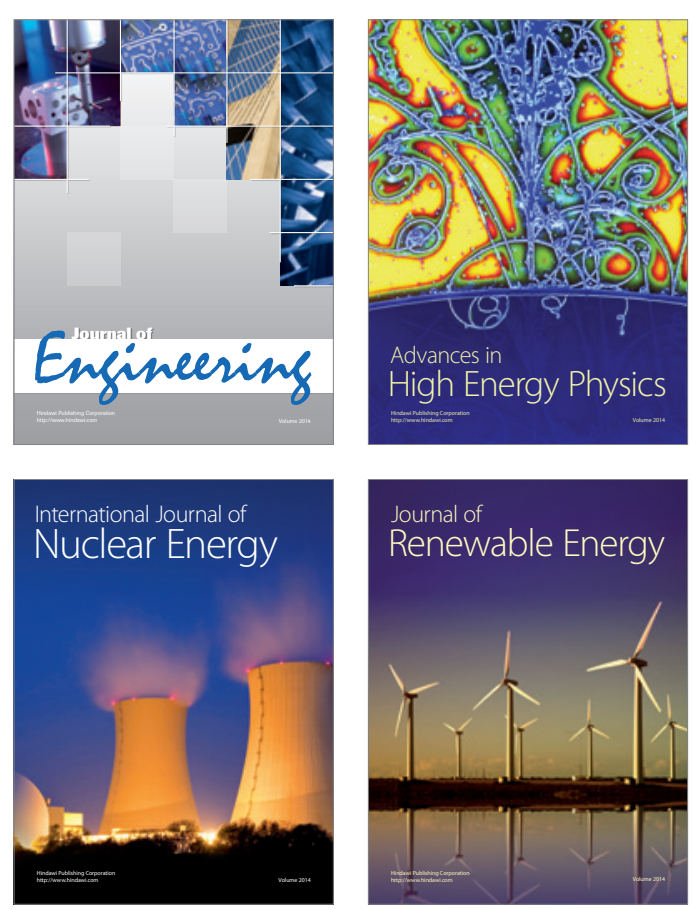

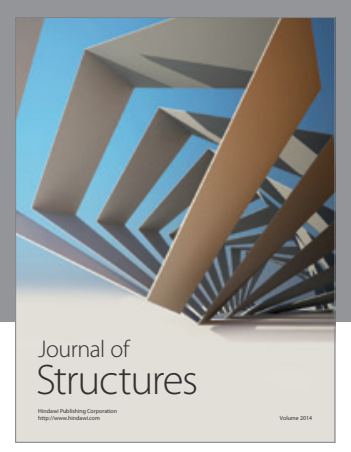

Rotating
Mechinery
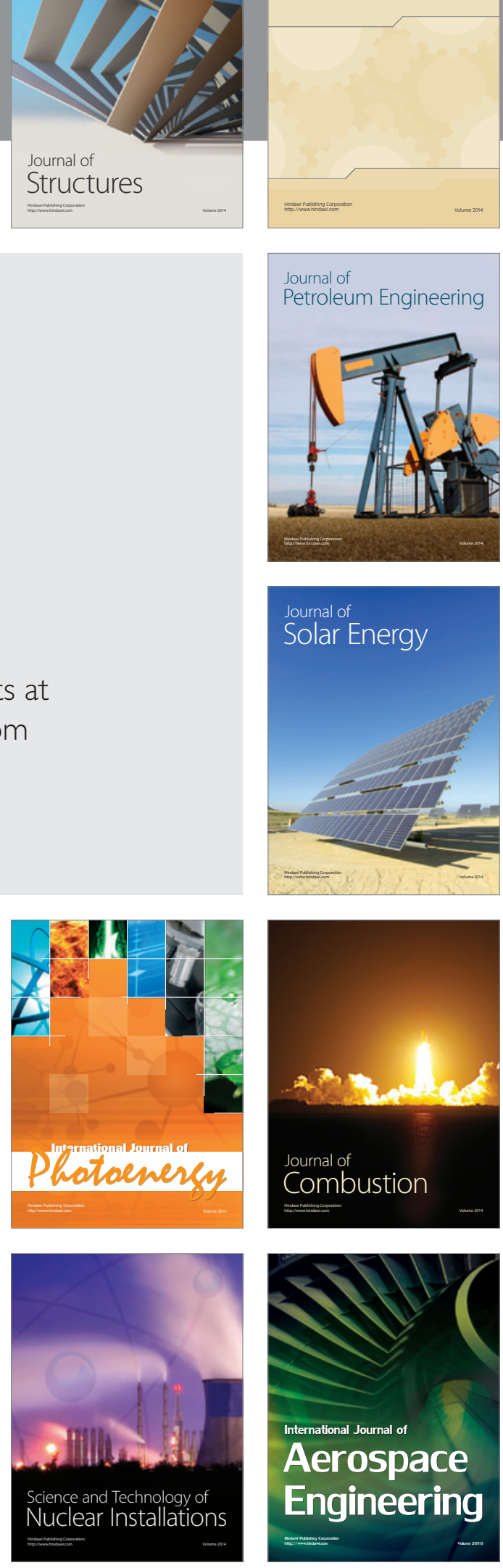